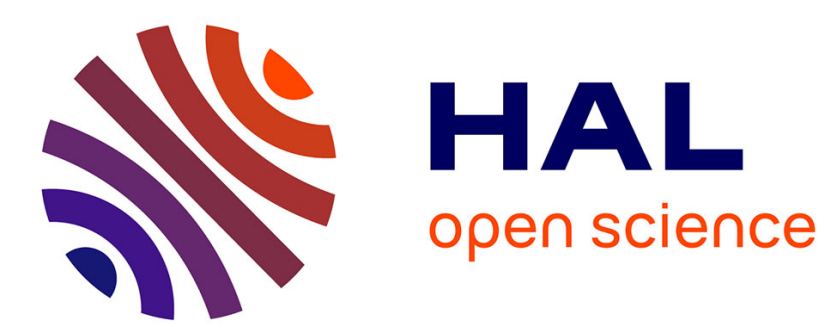

\title{
Optimization of Contrast Resolution by Genetic Algorithm in Ultrasound Tissue Harmonic Imaging
}

Sébastien Ménigot, Jean-Marc Girault

\section{To cite this version:}

Sébastien Ménigot, Jean-Marc Girault. Optimization of Contrast Resolution by Genetic Algorithm in Ultrasound Tissue Harmonic Imaging. Ultrasonics, 2016, 71, pp.231-244. 10.1016/j.ultras.2016.06.022 . hal-01340713

\section{HAL Id: hal-01340713 https://hal.science/hal-01340713}

Submitted on 1 Jul 2016

HAL is a multi-disciplinary open access archive for the deposit and dissemination of scientific research documents, whether they are published or not. The documents may come from teaching and research institutions in France or abroad, or from public or private research centers.
L'archive ouverte pluridisciplinaire HAL, est destinée au dépôt et à la diffusion de documents scientifiques de niveau recherche, publiés ou non, émanant des établissements d'enseignement et de recherche français ou étrangers, des laboratoires publics ou privés. 


\title{
Optimization of Contrast Resolution by Genetic Algorithm in Ultrasound Tissue Harmonic Imaging
}

\author{
Sébastien Ménigot ${ }^{\mathrm{a}, *}$, Jean-Marc Girault ${ }^{\mathrm{a}}$ \\ ${ }^{a}$ Université François-Rabelais de Tours, Inserm, Imagerie et Cerveau UMR U930, Tours, France
}

\begin{abstract}
The development of ultrasound imaging techniques such as pulse inversion has improved tissue harmonic imaging. Nevertheless, no recommendation has been made to date for the design of the waveform transmitted through the medium being explored. Our aim was therefore to find automatically the optimal "imaging" wave which maximized the contrast resolution without a priori information. To overcome assumption regarding the waveform, a genetic algorithm investigated the medium thanks to the transmission of stochastic "explorer" waves. Moreover, these stochastic signals could be constrained by the type of generator available (bipolar or arbitrary). To implement it, we changed the current pulse inversion imaging system by including feedback. Thus the method optimized the contrast resolution by adaptively selecting the samples of the excitation. In simulation, we benchmarked the contrast effectiveness of the best found transmitted stochastic commands and the usual fixed-frequency command. The optimization method converged quickly after around 300 iterations in the same optimal area. These results were confirmed experimentally. In the experimental case, the contrast resolution measured on a radiofrequency line could be improved by $6 \%$ with a bipolar generator and it could still increase by $15 \%$ with an arbitrary waveform generator.
\end{abstract}

Keywords: Genetic Algorithm, optimal command, pulse inversion, tissue harmonic imaging, stochastic signal, ultrasound.

\section{Highlights}

- The contrast resolution is automatically optimized for ultrasound harmonic image.

- Random excitations explore the medium and a genetic algorithm selects the best one.

- Features of electrical generator can be taking into account (bipolar or arbitrary).

- The contrast resolution reached an optimal value higher than with the usual case.

\footnotetext{
*Imagerie et Cerveau UMR U930, Université François-Rabelais de Tours, Polytech Tours, 7 avenue Marcel Dassault, 37200 Tours, France

Email addresses: sebastien.menigot@univ-tours.fr (Sébastien Ménigot), jean-marc.girault@univ-tours.fr (Jean-Marc Girault)
} 
- From the benchmark in simulation, the method was applied experimentally.

\section{Introduction}

Sensitivity in medical ultrasound imaging systems has been improved over the past twenty years in order to provide more accurate medical diagnoses. The waveform design has made possible the improvement about the contrast and/or of the spatial resolution in ultrasound image. The technology of multi-element ultrasound probe has made possible the first kind of waveform design, i.e. the beamforming [1] based on Radar network [2]. The second kind based directly on the time series transmitted has made possible the development of harmonic imaging techniques [3] based on the nonlinear propagation of the ultrasound wave in tissues under exploration. When ultrasound sinus waves of frequency $f_{0}$ propagate nonlinearly, the echoes received are composed of harmonic components $\left(2 f_{0}\right.$, $\left.3 f_{0}, \ldots\right)$. By extracting each harmonic component, it is possible to obtain ultrasound images with high contrast $[4,5]$. Given this success, tissue harmonic imaging has become the native imaging modality in conventional ultrasound scanners.

However, in order to improve detection of the harmonic components, a limited pulse bandwidth is required [6], thereby limiting the axial resolution. To limit these drawbacks, some methods of the second kind of waveform design add the encoding of the transmitted excitation based on the error detection. They hypothesize that the error between several copies of echoes is mainly composed of backscattered nonlinearities. Some discrete encoding techniques with multiple transmissions [7] such as pulse inversion imaging [8], power modulation [9], contrast pulse sequencing [10] and pulse subtraction intermodulation [11] have been thus made possible to ensure good axial resolution while increasing the contrast. Moreover, this principle has been extended to continuous encoding in order to solve the trade-off between resolution and penetration, as in chirp imaging [12]. Finally some solutions have mixed the two encoding types as coded tissue harmonic imaging with pulse inversion [13] or mixed with spatial waveform design [14].

In any event, whereas the beamforming can be obtained from an optimal and adaptive process [15], all previous solutions of the encoding excitations are non-optimal. The question of waveform design remains open. In most cases, the solution of the excitation adopted by manufacturers consists of providing pre-set transmit frequencies linked with the available probe. However, in our opinion, the settings of the excitation must take into account the targeted medium and they must derive from an optimal command process. In medical ultrasound imaging, the optimal command problem consists of seeking the optimal excitation $w^{\star}(n)$ which provides the best contrast resolution $C$ :

$$
\mathbf{w}^{\star}=\underset{\mathbf{w}}{\operatorname{argmax}}(C(\mathbf{w})),
$$

where $\mathbf{w}=\left[w(0), w(1), \ldots, w\left(N_{s}\right)\right]^{T}$ is the digital transmitted signal, ${ }^{T}$ the symbol of the vector transposition and $N_{s}$ the total number of samples.

The first solution proposed to solve this problem was to use the invariance properties of the wave propagation equation [16]. Time reversal imaging optimizes the signal-to-noise ratio thanks a matched filter and can thus cancel the nonlinear components [17]. Note 
that its main practical use in medical imaging today is limited to automatic focusing in HIFU [18].

To overcome this limitation, another interesting solution has recently been proposed in medical ultrasound contrast imaging [19] and in tissue harmonic imaging [20]. This novel method solves equation 1 by transforming shape optimization into parametric optimization. In the latter study, the parameter to be optimized was the transmit frequency in order to maximize the cost-function, i.e. contrast-to-tissue ratio $(C T R)$. The computation of this optimal frequency was obtained automatically by using a simple algorithm of the gradient descent [21]. The cost-function $C T R$ is thus maximized iteratively thanks to the gradient $\nabla_{f} C T R=\frac{\partial C T R}{\partial f}$. Although this method is simple and suboptimal, it lays the groundwork for the optimal command paradigm. However, it also requires an arbitrary waveform generator that increases both the complexity and the cost of ultrasound scanner. To limit both complexity and cost, unipolar (voltage impulse V) or bipolar (voltage $-\mathrm{V}$ or $\mathrm{V}$ ) generators are preferred in conventional ultrasound scanners. Within this framework, an interesting study dedicated to improving the signal-to-noise ratio was proposed to convert a nonbinary code in a binary code [22]. It might adjusted with the previous optimization methods but only for the binary generator. However, to free itself from the waveform assumption, the optimization of the CTR (between a tissue perfused with a contrast agent and non-perfused surrounding tissue) in ultrasound contrast imaging has been able to simulate from bipolar stochastic waves [23]. This stochastic method has shown the existence of a better command than the usual fixed-frequency waves by transmitted a nonlinear excitation. Nevertheless, no information about the robustness and the experimental application has been shown while adjusting the method according to the generator. Moreover, no equivalent satisfactory method has been proposed to date in tissue harmonic imaging to find the optimal excitation.

The study we report here is an extension of the previous work presented in [23] for application in tissue harmonic imaging. First, the cost-function have to be adjusted to tissue harmonic imaging. Second, several stochastic signals have to be considered (not only ternary signals) in order to take into account the generator feature. The aim of this study is therefore to find automatically the optimal command which maximizes the contrast resolution without a priori information and which is constrained by type of the generator available (eq. 1).

We have thus changed a current nonlinear imaging system into a new system including feedback based on the transmission of "smart" waves generated by either a binary generator or an arbitrary waveform generator. The optimization process that we have proposed is composed of two steps. The first consists in sending random "explorer waves" generated by a genetic algorithm to probe the medium. The second transmits an optimal "imaging wave" adjusted to the medium studied. The solution is in fact found automatically without a priori information about the waveform from the single echo analysis of these "explorer waves". Only, the assumption on the generator used is required, which guarantees that the method can adjust itself whatever the image construction (beamforming or nonlinear extraction).

In this study, we validated this method with a pulse inversion imaging system. First the waveform optimization for the contrast resolution was applied in simulations. We 
statistically benchmarked to determine the experimental conditions in order to apply the method. Secondly we confirmed the optimization results experimentally.

\section{Waveform Optimization}

As explained above, the optimal pulse inversion imaging system includes a feedback as described in Fig. 1 in order to transmit the optimal waveform $\mathbf{x}^{\star}$. The optimization process is split in two steps for each iteration $k$ : "exploring" and "imaging" phase.

[Figure 1 about here.]

\subsection{Stochastic "Explorer" Waves}

At the iteration $k$, the first step of the optimization process consists in "exploring" the medium. $M$ stochastic commands $\mathbf{w}_{k, m}$ are randomly drawn thanks to a genetic algorithm (switch on position 1 in Fig. 1). Thus for the $m$-th individual solutions, the stochastic signal $\mathbf{w}_{k, m}$ and its copy with opposite phase, make the "explorer" waves $\mathbf{x}_{k, m}$ :

$$
\left\{\begin{array}{l}
\mathbf{x}_{k, m}^{(+)}=A_{k, m} \cdot w_{k, m} \\
\mathbf{x}_{k, m}^{(-)}=-A_{k, m} \cdot w_{k, m}
\end{array}\right.
$$

where the stochastic commands $\mathbf{w}_{k, m}$ is only defined on $N_{S}$ samples such as $\mathbf{w}_{k, m}=$ $\left[w_{k, m}(0), w_{k, m}(1), \ldots, w_{k, m}\left(N_{S}-1\right)\right]^{T}$. They are sequentially transmitted to the pulse inversion imaging system with a delay $\Delta n$ (timeline on Fig. 1). This delay $\Delta n$ between the excitation is chosen in relation with the depth exploration $D$ as $\Delta n=4 \cdot D / c_{w}$ with $c_{w}$ the wave celerity in water. Note that the coefficient 4 is set as a function of the tradeoff between a high frequency of transmission/reception and a reception safety. Moreover the duration $T_{\text {pulse }}$ of the stochastic signal $\mathbf{w}_{k, m}$ corresponds to $100 \%$ of the fractional bandwidth of the transducer. Thus $N_{s}$ samples are set by the genetic algorithm.

Finally, to obtain a constant power of the pulse $\mathbf{x}_{k, m}$ equal to $P_{x_{r e f}}$ for all stochastic signals transmitted, the amplitude of the driving pressure is adjusted such as:

$$
A_{k, m}=\sqrt{\frac{A_{0}^{2} \cdot P_{\mathbf{x}_{r e f}}}{\mathbf{w}_{k, m}^{T} \cdot \mathbf{w}_{k, m}}}
$$

where the power $P_{x_{r e f}}$ is computed for a signal $\mathbf{x}_{r e f}$ which is the impulse response of the transducer with a driving pressure $A_{0}$. The power of the transmitted signal $x_{k, m}$ remained constant by adjusting the amplitude signal $A_{k, m}$. Note that the power is computed as the temporal average of squared signal.

\subsection{Contrast Resolution}

For each one of the $M$ individual solutions of the iteration $k$, the respective echoes are measured to construct $M$ radiofrequency lines $\mathbf{z}_{k, m}$, such as their samples $z_{k, m}(n)$ are defined as :

$$
z_{k, m}(n)=y_{k, m}^{(+)}(n)+y_{k, m}^{(-)}(n),
$$


where $\mathbf{y}_{k, m}^{(+)}$and $\mathbf{y}_{k, m}^{(-)}$are the radiofrequency obtained from $\mathbf{x}_{k, m}^{(+)}$and $\mathbf{x}_{k, m}^{(-)}$respectively. The radiofrequency line $\mathbf{z}_{k, m}$ is thus composed of harmonic components only and without any filtering [8]. Note that this property makes possible a good axial resolution while increasing the contrast.

The $M$ radiofrequency lines are therefore analysed to evaluate a usual contrast metric between two different tissues in tissue harmonic imaging. The cost function is thus defined as the contrast resolution $C_{k}$ between two areas containing two different tissues [24]. The two areas are delineated manually before the optimization process, but a segmentation step could be implemented to help the delineation process. However, if the medium has more than two kinds of tissue, the delineation has to choose a target among several tissues.

In this study, note that without loss of generality and for greater simplicity of the experimental setup, each contrast resolution $C_{k, m}$ was measured from only radiofrequency line, rather a whole image. However, in a full ultrasound imaging system, we supposed that the contrast resolution $C_{k, m}$ will estimate on the whole image and so limiting the transmitted intensity in the same area. Moreover, although the contrast resolution $C_{k, m}$ would be able to be computed from envelope amplitude related to the grey level image, a power computation can reveal the mean harmonic behaviour of each area for the radiofrequency line $\mathbf{z}_{k, m}$, while avoiding the envelope computation. Thus the contrast resolutions $C_{k, m}$ were defined as the normalized difference between the harmonic power $P_{1, m}$ backscattered by the area of the first tissue and the harmonic power $P_{2, m}$ backscattered by the area of the second tissue [24] as follows:

$$
C_{k, m}=\frac{P_{1, m}-P_{2, m}}{P_{1, m}+P_{2, m}}
$$

\section{3. "Imaging" Wave}

At the end of each iteration $k$, the second step consists in "imaging" the medium (switch on position 2 in on Fig. 1). The best individual solution among the "explorer" wave $\mathbf{x}_{k, m}$ can become the "imaging" wave $\mathbf{x}_{k}^{\star}$ such as:

$$
\mathbf{x}_{k}^{\star}=\underset{\substack{\mathbf{x}_{k, m} \\ m=1, \ldots, M}}{\operatorname{argmax}}\left[\mathbf{C}_{k, m}\left(\mathbf{x}_{k, m}\right)\right] .
$$

This "imaging waves" $\mathbf{x}_{k}^{\star}$ is sent to the pulse inversion imaging system in order to acquire the radiofrequency lines $\mathbf{z}_{k}^{\star}$. Several radiofrequency lines $\mathbf{z}_{k}^{\star}$ are usually juxtaposed for the process of image construction [1]. Finally the envelope of these $N_{l}$ respective echoes $\mathbf{z}_{k}^{\star}$ are computed to construct a grey level image from which the medical diagnosis can be obtained.

At this step, it should be noted that although our technique can offer an optimal stochastic command for each image line by maximizing a contrast resolution per line, the ideal excitation is transmitted for all lines making up the whole image to ensure the same axial resolution everywhere. Moreover, for low iteration number, the "imaging" phase could be stopped as long as the optimization process does not find a minimum contrast resolution desired $C_{m i n}$. In opposition, the "exploring" phase could be stopped as soon as the contrast resolution reach the contrast value desired $C_{\text {desired }}$. 


\subsection{Genetic Algorithm}

As previously explained, the search for the optimal command $\mathbf{w}^{\star}$ is based on the selection of the stochastic signals $\mathbf{w}_{k}$. However, finding the optimal imaging wave requires a large number of stochastic "explorer" signals from Monte-Carlo methods, since for example there are $2^{1 \cdot N_{s}}$ possibilities for a binary solution and $2^{12 \cdot N_{s}}$ possibilities for a discrete solution coded on 12 bits. We therefore proposed using metaheuristics to limit the search to best solutions and so reduce the computation time. Among metaheuristics, genetic algorithms can find the optimum by setting a vector containing parameters based on the principle of genetic reproduction $[25,26]$. This therefore led us to use a genetic algorithm where the vector was $N_{s}$ samples of the stochastic signal $\mathbf{w}_{k}$.

In the genetic algorithm described in Appendix $\mathrm{A}$ [27], $M$ initial individual stochastic solutions $\mathbf{w}_{0}$ are constructed at each iteration $k=0$. Two situations have been proposed to describe the stochastic signal samples $\mathbf{w}_{k, m}$ in order to take into account the constraints of the electrical signal generators. Each one of the $N_{s}$ samples is randomly chosen from (i) a discrete uniform distribution ( -1 or 1 ) for bipolar generators or (ii) a continuous uniform distribution between -1 and 1 for arbitrary waveform generators.

For the next generation $(k>0)$, only the $M / 2$ best individual solutions which maximize the contrast resolution are kept to become parents. To construct the offspring of $M / 2$ new individual solutions, the crossover operator mixes the best parent with one of the remaining $M / 2-1$ parents. Thus some of the first parent samples up to the crossover point and some of the second parent samples from the crossover point constitute the offspring. It should be noted that the crossover point is randomly selected between the first and the last sample. Moreover, samples are randomly changed according to the mutation rate $R_{M}$ in order to obtain robust optimization.

Before implementing the algorithm, the population size $M$ must be chosen. The tradeoff between robustness and the computation time due to acquisition of each individual solution requires a small population and a high mutation rate [27]. Moreover, the population size $M$ is link to the frame rate using the imaging wave so that suboptimal images can be shown regularly during the optimization process. Thus we propose that $M$ is a sub-multiple of the frame rate theoretically defined such as $f_{F R}=1 /\left(2 \cdot D / c_{w} \cdot N_{l}\right)$. For example, in this study, since the exploration depth was around $5 \mathrm{~cm}$, the theoretical maximal frame rate was around $120 \mathrm{~Hz}$. Note that the maximal population size can be around the highest frame rate conventionally used (i.e. around $100 \mathrm{~Hz}$ ). Therefore, the population size $M$ was at 12 with a theoretical frame rate of $10 \mathrm{~Hz}$ (during the optimization process), because the 12 cores of our processing unit allowed us to simulate the 12 individual solutions. Finally, the mutation rate $R_{M}$ is set to $40 \%$ as proposed in [27], because the number $M$ of individual solutions is small.

To summarize, several initial solutions are randomly selected. Then at each generation, only the best solutions which maximize the contrast resolution are kept. By a mechanism close to genetic reproduction, these best solutions are mixed to construct new solutions. However, to avoid a local optimization, some samples are modified randomly. Finally the suboptimal stochastic command $\mathbf{w}^{\star}$ is the best individual solution $\mathbf{w}_{\mathbf{k}}$ of generation $k$, which can reach the optimal stochastic command by increasing the iteration $k$. 


\section{Simulation Evaluation}

The optimization principle was initially applied in simulation. Several simulations were performed to demonstrate the feasibility of our method without any experimental constraints. In this section, after the presentation of the simulation model, we study the robustness of the optimization convergence. Finally, we show the features of the best found solutions.

\subsection{Simulation Model}

In order to validate the feasibility, we used realistic simulations which have already been proven in medical ultrasound imaging $[19,28]$. The simulation model is constructed on the basis of the pulse inversion imaging system (Fig. 1). It is composed of different phases: transmission, 2D nonlinear propagation and reception.

\section{[Table 1 about here.]}

At the transmitter, a stochastic signal $\mathbf{x}_{k, m}$ is generated digitally with Matlab (The Mathworks, Natick, MA, USA). It is transmitted to a single-element probe with a diameter of $1 \mathrm{~mm}$ and filtered by its transfer function from a realistic transducer, centred at $f_{c}=4 \mathrm{MHz}$ with a fractional bandwidth of $80 \%$ at $-3 \mathrm{~dB}$. The pulse wave generated is propagated nonlinearly into an attenuating medium, i.e. a $5 \mathrm{~mm}$-broad inclusion of a second tissue sample is inserted into the first tissue at $9.5 \mathrm{~mm}$ below the surface (Fig. 2 ). Note that these small sizes were chosen to reduce the computation time of the propagation simulation. The two different tissues are selected to mimic two real materials (blood and fat) with very different properties described in Table 1 . The 2D nonlinear wave propagation is solved using the Anderson model based on a pseudo-spectral derivative and a time-domain integration algorithm [29]. This solver requires three grids: a grid of mean density, a grid of mean speed of sound and a grid of a $B / A$ nonlinearity parameter described in Table 1 . The scatterers are generated randomly by weakly modifiying the density grid and the velocity grid. Moreover this model included an attenuation of $0.5 \mathrm{~dB} /(\mathrm{MHz} \cdot \mathrm{cm})$. Note that this attenuation was constant in the medium in order to simplify the simulation model.

[Figure 2 about here.]

Finally, the signals backscattered by tissue are recorded. These tissue echoes are filtered by the transfer function of the transducer to construct the echoes. Then the radiofrequency line $\mathbf{z}_{k, m}$ is constructed from equation 4 . An example of a radiofrequency line is depicted in Fig. 2. The contrast resolution $C_{k, m}$ is thus computed from the radiofrequency line $\mathbf{z}_{k, m}$ and the optimization process computes a new stochastic signal $\mathbf{x}_{k, m}$ to explore the medium again. Finally, it is possible to build an image at the end of each generation by moving the transducer. 


\subsection{Statistics of Contrast Optimization}

Contrast optimization was applied to the previous simulation model for two different transmitters (bipolar and arbitrary waveform). In order to demonstrate the robustness and the reproducibility of the method, we propose to compute the contrast resolution statistically from several optimization. The optimization was thus repeated 20 times with different random initialization. As the method required a high frame rate, a problem of tissue safety can appear and can be solved by reducing the mechanical index (MI). It has been shown that tissue harmonic imaging could be performed with low MI [30]. For each simulation, the driving pressure $A_{0}$ was set at $200 \mathrm{kPa}$, and thus the MI was always low around 0.1. Moreover, the stochastic signal had a duration $T$ which represented $100 \%$ of the fractional bandwidth of the transducer. According to the sampling rate $\left(f_{s}=46 \mathrm{MHz}\right)$ of the simulation model, 40 samples were required in duration $T_{\text {pulse }}=$ $0.9 \mu \mathrm{s}$, and therefore $N_{s}=40$. Moreover, to demonstrate the effectiveness of the new method, the results were compared to those of two usual transmitted Gaussian-modulated sinusoidal pulses $[31,8]$. They have the same bandwidth and the same driving pressure than the stochastic signals. Their transmit frequencies were set at (i) two-thirds of the central frequency $f_{c}$ of the transducer [31] $\left(2 / 3 f_{c}=2.67 \mathrm{MHz}\right)$, and (ii) the optimal fixed-frequency $f^{\star}=1.9 \mathrm{MHz}$. Note that this best fixed-frequency $f^{\star}$ made it possible to obtain a limited contrast resolution $C_{f^{\star}}$ which was the best solution by selecting the transmit frequency only [19].

[Figure 3 about here.]

Fig. 3 shows the statistics of the contrast resolution $C$ as a function of generation $k$ in binary and non-binary situations. These distributions are represented with their median and their four quartiles. As an illustration, these results are compared with the two fixed-frequency signals.

By using frequency optimization (red dotted line in Fig. 3), it was possible to achieve a suboptimal solution which could offer a gain of $15 \%$ in comparison with contrast obtained by the signal transmitted at the usual transmit frequency (cyan dotted line in Fig. 3). However, the contrast resolution $C$ was higher with the two best found stochastic commands than with the usual transmit frequency. These contrast resolution values could not be achieved with the fixed-frequency signals, although the transmit frequency was optimized. After 3217 generations in the binary optimization, the contrast resolution $C$ reached a median best found value of $80 \%$ that was statistically higher than the frequency setting situations. Moreover, by using a continuous genetic algorithm and an analogue generator, the best found non-binary stochastic command considerably still improved the contrast resolution $C$, i.e. $87 \%$, after 3576 iterations. For instance, when the binary optimization was over (after 5000 generations), all the contrast resolution measured were between $79 \%$ and $81 \%$, whereas they were between $87 \%$ and $89 \%$ at the end of the nonbinary optimization. However, although it was not required to wait for a large number of generation to reach a contrast value close to the optimal contrast resolution, the number of generations was reduced to 300. This leads to a good trade off between a good contrast resolution and a reasonable duration. Moreover, a single generation using non-binary 
stochastic commands was sufficient to obtain a contrast resolution higher (contrast resolution between $72 \%$ and $80 \%$ ) than this one by using the optimal fixed-frequency. By using binary stochastic command, seven generations was necessary to obtain the same property (contrast resolution between $68 \%$ and $75 \%$ ), because of the limitation of degrees of freedom.

To summarize, with the best found binary stochastic command, the contrast resolution could be increased to $27 \%$ on average compared to the situation with the usual fixedfrequency transmitted signal. When the number of degrees of freedom was high, it could be still increased up to $8 \%$ on average compared to the situation of the transmitted signal at the optimal fixed-frequency $f^{\star}$.

[Figure 4 about here.]

[Figure 5 about here.]

Fig. 4 and Fig. 5 show the input and output signals for the best "imaging" signals when the command was (i) the best found binary stochastic signal and (ii) the best found non-binary stochastic signal $\mathbf{w}^{\star}$, respectively.

The best found binary and non-binary commands are depicted in Fig. 4a and Fig. 5 a respectively. The lines show the median of the sample value and the areas show the quartiles from the twenty binary and non-binary optimizations. In others words, a median excitation appeared in between the quartile curves either for the binary command or for the non-binary command. The optimization may reach the same optimal region including the same optimal value without reaching it accurately. Note that this drawback is a metaheuristics property which can be avoid by adding a local optimization algorithm [32], but with the risk to increase the iteration number.

Fig. 4c and Fig. 5c show the waves at the transducer output transmitted to the tissue when the stochatic command $\mathbf{w}$ was the best found binary and non-binary signals respectively. As expected, the transmitted wave were no more discrete nature due to the filtering effects of the transducers. Moreover, because of the genetic algorithm, all best found stochastic commands $\mathbf{w}$ and best found waves were similar, since they were confined to a small range around the mean best found "imaging" wave (blue and green areas in Fig. 4c and Fig. 5c).

The respective spectra of these best found "imaging" waves and the transducer bandwidth are depicted in Fig. 4b and Fig. 5b respectively. As an illustration, the results are compared with a Gaussian-modulated sinusoidal pulses at the optimal fixed transmit frequency $f^{\star}$. Note that this optimal fixed-frequency wave transmit has been filtered by the transducer, which explains the shape of its spectrum and its central frequency. As observed in [23], the best found "imaging" waves had several frequency components in the transducer bandwidth, unlike a usual fixed-frequency transmitted wave. Although the best found "imaging" wave was composed of the optimal fixed-frequency transmitted wave, the amplitude of this fundamental frequency component were reduced to distribute the power on other frequency components, in particularly around three times the lower transmit frequency ( 2 to $6 \mathrm{MHz}$ ). These added frequency components were more easily recognizable in the non-binary case than in the binary case. 
Finally Fig. 4e and Fig. 5e show the respective radiofrequency lines, and their respective spectra are presented in Fig. 4d and Fig. 5d. Note that for more clearness, only the median radiofrequency lines were depicted on Fig. 4e and Fig. 5e. The radiofrequency line was composed only of the nonlinear components backscattered by the tissue remained, because the linear component was suppressed in pulse inversion imaging (detailed explanation in Appendix Appendix B). Even if the backscattered second harmonic components of these higher transmit frequencies were not present in the radiofrequency lines, the contrast resolution could not reach the optimal value without that transmit frequencies. Therefore the nonlinear backscattered components were the sum of (i) the second harmonic components which were the double lower frequency of the components transmitted and (ii) the intermodulation components between the lower and higher transmitted frequency.

\section{[Figure 6 about here.]}

In order to clearly see the contrast resolution on the radiofrequency line, Fig. 6 shows the short-term harmonic powers of the radiofrequency lines when the signals transmitted were the best found non-binary stochastic signal, the best found binary stochastic command and the two fixed-frequency signals. Moreover, the blue and green areas show the statistical distribution of the harmonic short-term mean powers for the twenty binary and non-binary optimizations. As the radiofrequency lines shown in Fig. 6, the backscattered harmonic power increased with the inclusion of the second tissue, whatever the transmitted signal. However, as can be seen from the results presented in Fig. 3, the ratio of the harmonic short-term mean powers between the two tissues was greater with the best found stochastic commands. Moreover, although the first quartile of the short-term harmonic power from non-binary command was overlapped with the short-term harmonic power from binary command, three quarters of the short-term harmonic power from non-binary command were close to its median value which was higher than the distribution from the binary command. The most complex waveform of the non-binary command may explain the difference between the distribution.

\section{[Figure 7 about here.]}

Finally, as an illustration of the second step with "imaging waves", Fig. 7 shows the synthetic image using the best best found non-binary stochastic signal, the best best found binary stochastic command, the two fixed-frequency signals and their respective contrast resolution $C$. Optimization was performed for the central radiofrequency line of the region of interest and the same transmitted signal was applied for all the radiofrequency lines of the image. However the images show the same increase in the contrast $C$ between the two tissues as the values measured only on the center radiofrequency lines shown in Fig. 3. Note that the differences between the contrast measured on one line and the whole image must be due to the random position of scatterers. As can be seen from the results presented in Fig. 4 and Fig. 5, the signal backscattered by the second tissue was lower in the two images using the usual transmitted signal than in the images using an best found stochastic command. Moreover, the speckle seemed to be thin by using the non-binary command, since the distribution of the short-term harmonic power showed a larger range for the low value. 


\section{Experimental Validation}

The aim of this section was to experimentally confirm the optimization results obtained in the simulations.

\subsection{Experimental Setup}

The experimental setup follows the steps presented in Fig. 8. It makes possible to have a pulse-echo system.

[Figure 8 about here.]

The stochastic signals $\mathbf{x}_{k, m}$ are generated digitally by Matlab with a personal computer (PC). They are successively transmitted to an 12-bits arbitrary waveform generator (PicoScope PS5203, PicoTech, St Neots, UK) by USB. They are then amplified by $50 \mathrm{~dB}$ using a power amplifier (AAP-200-1-10, Adece, Veigné, France) protected by a 3 dB high power attenuator (HFP-5100-3/3-NM/NF, Trilithic, Indianapolis, IN, USA). Finally they are transmitted to the medium from a 3.3 MHz PZT single element transducer (V380-SU, Olympus NDT Inc., Waltham, MA, USA) with a bandwidth of $64 \%$ at $-6 \mathrm{~dB}$. Note that the pulse repetition frequency of $2 \mathrm{kHz}$ is controlled by an outside clock device (Ultrasound receiver, GIP Ultrasons, Tours, France).

The wave propagates through a tissue-mimicking phantom (model 054GS, General Purpose Ultrasound Phantom, CIRS, Norfolk, VA, USA). This phantom is made of Zerbine polymer [33], where the attenuating coefficient is $0.5 \mathrm{~dB} / \mathrm{cm}-\mathrm{MHz}$ and the speed of sound is $1540 \mathrm{~m} / \mathrm{s} \pm 10 \mathrm{~m} / \mathrm{s}$. It includes a $8 \mathrm{~mm}$-broad hyperechoic inclusion at a 40 mm-depth. Note that the studied area is close to the grid shown in Fig. 2. The backscattered waves are collected by the same transducer. The echoes $\mathbf{y}_{k, m}$ are transmitted to an ultrasound receiver via a diode bridge and amplified by $40 \mathrm{~dB}$. Finally their measurements are repeated by an oscilloscope (PicoScope PS5203) four times to increase the signal-tonoise ratio. From these four measurements, the PC constructs a mean pulse inversion

radiofrequency line $\mathbf{z}_{k, m}$ before selecting the next stochastic signal $\mathbf{x}_{k+1, m}$ at the iteration $k+1$.

After the optimization was performed for the central radiofrequency line of the region of interest, the acoustic pressure of the best found transmitted "imaging" waves were measured with a 85- $\mu \mathrm{m}$ hydrophone (HGL0085, ONDA Corp., Sunnyvale, CA) close to the transducer. Finally, the same "imaging" signal was applied for all the image lines of the image. These other lines was obtained by shifting the transducer thanks to a motorized positioning systems (Owis DC-500, OWIS GmbH, Staufen, Germany) by step of $0.25 \mathrm{~mm}$. Note that the phantom position is in the longitudinal management in order to facilitate the probe displacement.

\subsection{Experimental Results}

[Figure 9 about here.]

The experimental results are presented in Fig. 9 for a single optimization.

Fig. 9a shows the best contrast resolution $C$ as a function of generation $k$ in binary and non-binary situations. As an illustration, these results are compared with the two 
fixed-frequency signals. Because of the memory of the experimental device, the optimizations were stopped after 330 generations which had to come closer to the convergence according to the simulation (Fig. 3). These results were in accordance with our simulation results: we observed that the best found stochastic command made possible to increase the contrast resolution $C$ in comparison with the frequency setting situations. Note that the difference between the contrast resolution $C$ for the frequency setting situations in our simulation and that obtained in our experiment might be due to the noise on measurements higher in experiment than in simulation. It explained the difference of contrast gain, because the reference was not the same. Therefore, by using frequency optimization, it was possible to achieve a suboptimal solution which could offer a gain of $6 \%$ in comparison with contrast obtained by the signal transmitted at the usual transmit frequency. However, the contrast resolution could be increased to $11 \%$ by using best found binary command compared to the situation with the usual fixed-frequency transmitted signal. It could be increased to $17.5 \%$ by using best found non-binary command. Finally, as in simulation, only one generation for the non-binary stochastic command and seven generations for the binary stochastic command was required. They enabled to reach a contrast resolution higher than this one obtained by using the optimal fixed frequency.

As an illustration of the step of the "imaging wave", Fig. 9b and Fig. 9c shows the best found non-binary stochastic command and the best found binary stochastic command $w^{\star}(n)$. Fig. 9d and Fig. 9e show the respective driving pressure at the transducer output transmitted to the tissue. Fig. 9e and Fig. 9h show the radiofrequency lines for the two different kinds of best found stochastic command and the two fixed-frequency signals. The amplitudes were higher with the two best found stochastic commands. This result was in accordance with the harmonic short-term mean power shown in Fig. 9j. As in the simulation, even if the harmonic short-term mean power could slightly increased with the best found stochastic commands, the ratio of the harmonic short-term mean powers between the two tissues was greater with the best found stochastic commands. Finally, Fig. 9f and Fig. 9g show the spectra of the transmitted and the backscattered waves for the best found binary and non-binary commands respectively. The transducer bandwidth is added on these spectra. The transmitted waves have a high frequency components, even if their amplitude was lower than in simulation. It can be due to difference of the bandwidth of the wave generation system, especially the transducer. Moreover, all these components were in the bandwidth transducer including the high components. These high components were not generated by nonlinearities from electronics, since the difference between the two transmitted signals in opposite phases was low.

[Figure 10 about here.]

Furthermore, as an illustration, Fig. 10 shows the experimental images using the best found non-binary stochastic signal, the best found binary stochastic command, the two fixed-frequency signals and their respective contrast resolution $C$. The experimental images show an increase of the contrast resolution $C$ between the two tissues, which is close to the previous results. The contrasts measured on the whole image were sightly lower than the ones measured on the center radiofrequency lines, because the scatterer distribution must be due different according the lines. Moreover, note that the difference 
between the images in simulations and in experiment can have several origins. First, the radiofrequency line could be blurred by the noise measurements higher in experiment than in simulation. Second, some tissue-mimicking properties might be different between in simulation and in experiment. Indeed, some data like $B / A$ parameter were not specified by the manufacturer. The image textures could therefore be different. Third, the experimental setup did not allow us to centre the inclusion in the image. The second part of the tissue 1 was bigger than the first part. Therefore the optimization process gave priority to reduce the contribution of the second part rather the first part, which explains that the first part of the tissue 1 was not as dark as in simulation and as the second part.

Finally, to summarize, these experimental results confirmed the feasibility of our method.

\section{Discussion}

Automatic optimization of contrast resolution $C$ for a pulse inversion imaging system was performed by stochastic sequences. Although the previous method [23] only gave the existence of a such optimal solution, robustness and speed was not demonstrated with the aim of applying it experimentally.

The closed loop system automatically provided an best stochastic command to achieve the optimal area of the contrast resolution $C$. According to the constraint on the command and because of the genetic algorithm, these optimal contrast resolutions were always closed each other and the best signals transmitted also had strong similarities. Even if it is well known that the the genetic algorithm can only found the area of the optimum, the algorithm thus converged to the same optimal contrast resolution whatever the initialization. Moreover this contrast resolution $C$ was higher than with usual Gaussianmodulated sinusoidal pulses at a fixed transmit frequency. All best found "imaging" waves were filtered stochastic commands composed of frequency components transmitted to the medium being explored. Without these different transmit frequency components, the excitation was a non-optimal fixed-frequency signal, even if the lower transmit frequency components were closed to the optimal fixed-frequency. The optimization must change the frequency distribution of the transmitted power, in order to facilitate the reception of the backscattered harmonic components. The power lost was therefore limited in comparison with the conventional methods. Moreover, the resulting image was a harmonic image which was not due to the direct transmission of harmonic components but to the harmonic components from the nonlinear interaction between the excitation and the medium. Note that only the discrete encoding techniques, e.g. the pulse inversion, can guarantee it (Appendix B). By taking into account the transducer bandwidth, the second harmonic components of the higher transmit frequencies generated by the nonlinear propagation were not directly used to construct the second harmonic image. The lower frequency components enabled to generate the backscattered second harmonic components, whereas the higher transmit frequency associated to the lower frequency amplified the generation of backscattered harmonic power by intermodulation.

Even if the frequency content of best found excitation was always complex, the number of degrees of freedom was not the same according to the specific features of electrical signal generators (bipolar generator or arbitrary waveform generator). The genetic algorithm 
took into account this choice by changing the random drawing of the sample excitation. The continuous genetic algorithm with a arbitrary waveform transmitter increased the number of degrees of freedom compared to using a binary genetic algorithm and a bipolar generator. Therefore, the higher its number of degrees of freedom was, the higher the contrast resolution could reach.

This transmitted stochastic signals adjusted itself for any medium explored according to this constraint applied to the best found waveform (binary or non-binary). The only information required about the medium was the position of the target being observed. No another a priori information about the medium nor about the transducer was required for this optimization, because the explorer waves gave informations to the cost function thanks to the mean power of the output system. Therefore it took into account the effects of attenuation and nonlinear propagation indirectly. Moreover, the cost-function defined as the contrast resolution was a tradeoff between increasing the harmonic power of the targeted tissue and decreasing the harmonic power of the surrounding tissue. Even if these two harmonic powers increased, the transmitted power was more suitable to the hyperechoic area, since the optimization reached a solution for which the ratio of the harmonic powers between the two tissues was bigger. Thus the optimization had to highlight hyperechoic area compared to the surrounding medium, whatever its depth. Note that according to the depth of the target, the optimization process will adjust the command. However, the surrounding medium included in the cost function should be equally distributed around the region of interest, so that no part of the surrounding is more important in the cost function.

Finally, the optimization duration can be crucial for future integration in an ultrasound imaging system. The minimum contrast resolution desired and the maximal frame rate of the imaging system are the main factors impacting the optimization duration. Indeed, the optimization duration is proportional to the "generation number required to reach the contrast resolution desired" $\times$ "the number of individual solution per generation $M$ " / "the frame rate of the system". Therefore these three factors can change the optimization duration. First, the high number of generations to reach the optimal contrast resolution may be a limiting factor. Nevertheless, the convergence could be reach quickly. Moreover a short optimization can be sufficient to obtain an interesting suboptimal solution. For instance, only one generation using non-binary stochastic pulses, i.e. twelve pulses, was required to obtain contrast resolutions higher than using usual Gaussian-modulated sinusoidal pulses. The contrast gain could be doubled by waiting for just more six pulses compared to the optimization of the single frequency. Moreover, in the case where we want to achieve the specific optimal contrast resolution, we linked the size population $M$ to the frame rate so that it is possible to show suboptimal image during the optimization process. Second, if the optimization settings are fixed, the only factor is the frame rate of the system. By assuming a sufficient frame rate, we could assume that the medium is quasi-static. The easiest solution would be to use a ultrafast imaging system. Therefore the definition of the cost function could remains constant whatever the low displacement caused by breathing for instance. Consequently, the comparison between the conventional methods and our method should be limited to the static tissue due to the frame rate. However, we though that our method would be more appropriate with a ultrafast 
imaging system. As a such system required a spatial compound with several angles, the optimization process may be used instead this average completely or partially. To summarize, we think that our system should be more suitable to an ultrafast imaging system in order to overcome the tissue displacement and to avoid the high local transmitted power (without focusing). However, more studies will necessary for the clinical applicability. For instance, because of the spatial-peak temporal-average intensity (ISPTA), the imaging of foetus or eyes should be excluded for now.

\section{Conclusions}

To conclude, we demonstrated that the usual imaging system was far from the optimal contrast resolution. With simple frequency optimization, it was possible to improve performance. However, to be close to the optimal performance, a bipolar generator of stochastic sequences was at least required, but only the generator of stochastic sequences gives the best performance.

Moreover, the optimization of the ultrasound wave transmitted through the medium had to take into account the full imaging system, from the transducer to electronic reception through the medium explored. It was therefore not possible to optimize a waveform once and for all, but required the optimization process to be performed for each tissue explored. In comparison with the conventional methods, even if the system can become more complex and can reduce the frame rate, the optimization was robust and can reach higher contrast resolution quickly. For example, such optimization might be performed after the clinician has chosen the cross section view.

\section{Acknowledgments}

We thank J.-M. Grégoire (Université François-Rabelais de Tours, Inserm, Imagerie et Cerveau UMR U930, Tours, France) for his advice in the conception of the experimental setup. We also want to thank D. Gross (Université François-Rabelais de Tours, GREMAN UMR-CNRS 7347, Tours, France) for his help to hydrophone measurements.

\section{References}

[1] D. H. Johnson, D. E. Dudgeon, Array Signal Processing: Concepts and Techniques, Simon \& Schuster, New York, NY, USA, 1992.

[2] K. W. Forsythe, D. W. Bliss, MIMO Radar: Concepts, Performance Enhancements, and Applications, John Wiley \& Sons, Inc., 2008, Ch. 2, pp. 65-121.

[3] F. Tranquart, N. Grenier, V. Eder, L. Pourcelot, Clinical use of ultrasound tissue harmonic imaging, Ultrasound Med Biol 25 (6) (1999) 889-894.

[4] P. N. Burns, Instrumentation for contrast echocardiography, Echocardiogr-J Card 19 (3) (2002) 241-258.

[5] Q. Ma, X. Gong, D. Zhang, Third order harmonic imaging for biological tissues using three phase-coded pulses, Ultrasonics 44, Supplement (2006) e61-e65. 
[6] M. A. Averkiou, Tissue harmonic imaging, in: Proc IEEE Ultrason Symp, Vol. 2, San Juan, Puerto Rico, 2000, pp. 1563-1572.

[7] F. Lin, C. Cachard, F. Varray, O. Basset, Generalization of multipulse transmission techniques for ultrasound imaging, Ultrason Imaging 37 (4) (2015) 294-311.

[8] Q. Ma, Y. Ma, X. Gong, D. Zhang, Improvement of tissue harmonic imaging using the pulse-inversion technique, Ultrasound Med Biol 31 (7) (2005) 889-894.

[9] G. A. Brock-fisher, M. D. Poland, P. G. Rafter, Means for increasing sensitivity in non-linear ultrasound imaging systems, US Patent 5,577,505.

[10] L. Thomas, S. Maslak, P. Phillips, G. Holley, Medical diagnostic ultrasound system using contrast pulse sequence imaging, US Patent 6,494,841.

[11] A. Nowicki, J. Wójcik, W. Secomski, Harmonic imaging using multitone nonlinear coding, Ultrasound in Medicine \& Biology 33 (7) (2007) 1112-1122.

[12] T. Misaridis, J. A. Jensen, Use of modulated excitation signals in medical ultrasound. part ii: Design and performance for medical imaging applications, IEEE T Ultrason Ferr 52 (2) (2005) 192-207.

[13] J. Song, S. Kim, H. Sohn, T. Song, Y. M. Yoo, Coded excitation for ultrasound tissue harmonic imaging, Ultrasonics 50 (6) (2010) 613-619.

[14] S. A. Izadi, A. Mahloojifar, B. M. Asl, Weighted capon beamformer combined with coded excitation in ultrasound imaging, Journal of Medical Ultrasonics 42 (4) (2015) $477-488$.

[15] J. F. Synnevag, A. Austeng, S. Holm, Adaptive beamforming applied to medical ultrasound imaging, IEEE Transactions on Ultrasonics Ferroelectrics and Frequency Control 54 (8) (2007) 1606-1613.

[16] M. Fink, Time-reversal of ultrasonic fields .1. basic principles, IEEE T Ultrason Ferr 39 (5) (1992) 555-566.

[17] O. Couture, J. F. Aubry, G. Montaldo, M. Tanter, M. Fink, Suppression of tissue harmonics for pulse-inversion contrast imaging using time reversal, Physics in medicine and biology 53 (19) (2008) 5469-5480.

[18] J.-L. Thomas, M. Fink, Ultrasonic beam focusing through tissue inhomogeneities with a time reversal mirror: application to transskull therapy, IEEE T Ultrason Ferr 43 (6) (1996) 1122-1129.

[19] S. Ménigot, J.-M. Girault, I. Voicu, A. Novell, Optimization of contrast to tissue ratio by frequency adaptation in pulse inversion imaging, IEEE T Ultrason Ferr 59 (11) (2012) 2431-2438. 
[20] S. Ménigot, J.-M. Girault, Optimal control by transmit frequency in tissue harmonic imaging, in: Acoustics 2012, Nantes, France, 2012, pp. 2863-2868.

[21] B. Widrow, S. Stearns, Adaptive Signal Processing, Prentice Hall, Englewood Cliffs, USA, 1985.

[22] S.-W. Huang, P.-C. Li, Arbitrary waveform coded excitation using bipolar square wave pulsers in medical ultrasound, IEEE T Ultrason Ferr 53 (1) (2006) 106-116.

[23] S. Ménigot, J.-M. Girault, Optimization of contrast-to-tissue ratio by adaptation of transmitted ternary signal in ultrasound pulse inversion imaging, Comput Math Meth Med 2013 (2013) 6.

[24] T. Szabo, Diagnostic Ultrasound Imaging: Inside Out, Academic Press, London, UK, 2004.

[25] J. H. Holland, Adaptation in Natural and Artificial System, The University of Michigan Press, Ann Arbor, MI, USA, 1975.

[26] J. Dréo, A. Pétrowski, P. Siarry, E. Taillard, Metaheuristics for Hard Optimization: Methods and Case Studies, 1st Edition, Springer, Heidelberg, Germany, 2006.

[27] R. L. Haupt, S. E. Haupt, Practical Genetic Algorithms, 2nd Edition, John Wiley \& Sons Inc, Hoboken, NJ, USA, 2004.

[28] T. Varslot, G. Taraldsen, Computer simulation of forward wave propagation in soft tissue, IEEE T Ultrason Ferr 52 (9) (2005) 1473-1482.

[29] M. E. Anderson, A 2d nonlinear wave propagation solver written in open-source matlab code, in: Proceeding IEEE Ultrasonic Symposium, San Juan, Puerto Rico, 2000, pp. 1351-1354.

[30] R. Lang, S. A. Goldstein, I. Kronzon, B. K. Khandheria, V. Mor-Avi, ASE's Comprehensive Echocardiography, 2nd Edition, Elsevier, Philadelphia, PA, USA, 2016.

[31] J. A. Hossack, P. Mauchamp, L. Ratsimandresy, A high bandwidth transducer optimized for harmonic imaging, in: Proc IEEE Ultrason Symp, Vol. 2, San Juan, Puerto Rico, 2000, pp. 1021-1024.

[32] R. Chelouah, P. Siarry, Genetic and nelder-mead algorithms hybridized for a more accurate global optimization of continuous multiminima functions, European journal of operational research 148 (2) (2003) 335-348.

[33] M. B. Zerhouni, M. Rachedine, Ultrasonic calibration material and method, US Patent 5,196,343. 


\section{Appendix A. Algorithm}

Result: Find the optimal stochastic signal $\mathbf{w}^{\star}$ to optimize the contrast resolution C

begin

\section{Initialization}

Choose $M$ initial stochastic signals $\mathbf{w}_{0, m}$ randomly;

for $i=1$ to $M$ do

ContrastEvalutation $\left(\mathbf{w}_{0, i}\right)$;

end

end

while $k<$ Maximal Iteration do

Sort the individual stochastic signals $\mathbf{w}_{k, m}$ in descending order of the contrast resolution $C$;

Keep the $M / 2$ best stochastic signals;

Generate $M / 2$ new individual stochastic signals by crossover ; // Mating

Change $40 \%$ samples of the new individual randomly; // Mutation

Replace the $M / 2$ worst individual stochastic signals by the new previous signal;

for $i=1$ to $M$ do

| ContrastEvalutation $\left(\mathbf{w}_{k, i}\right)$;

end

$\mathbf{w}^{\star} \leftarrow \mathbf{w}_{k, 1}$

$k \leftarrow k+1$

end

end

Function ContrastEvalutation(w) is

Result: Contrast resolution $C$

Construct the signal $\mathrm{x}$ from $\mathbf{w}$;

Transmit the signal $\mathbf{x}$ in the ultrasound system;

// described by eq. 2

Receive the echoes $\mathbf{y}$;

Receive the harmonic signal $\mathbf{z}$;

;

// described by eq. 4

Compute the harmonic power of the tissues 1 and 2 ;

Compute the contrast resolution $C ; \quad / /$ described by eq. 5 end

Algorithm 1: Pseudo-code of the contrast resolution maximization by genetic algorithm 


\section{Appendix B. Nonlinearity Extraction in Pulse Inversion Imaging}

The pulse inversion method can only extract the harmonic components from the nonlinear interaction between the excitation and the medium, whatever the signal transmitted. Using a simple example, we can demonstrate this principle.

First, the transmitted signal $\mathbf{x}$ can be cut in two parts composed each one of two components such as:

$$
\left\{\begin{array}{l}
x_{1}(n)=x_{11}(n)+x_{12}(n) \\
x_{2}(n)=x_{21}(n)+x_{22}(n)
\end{array}\right.
$$

where the components $x_{11}(n)$ and $x_{21}(n)$ correspond to the fundamental components at $f_{0}$, and the components $x_{12}(n)$ and $x_{22}(n)$ correspond to the third harmonic components at $3 f_{0}$. However, in pulse inversion imaging, the two transmitted signals are in opposite phase, i.e. $-x_{1}(n)=x_{2}(n)$; therefore $-x_{11}(n)=x_{21}(n)$ and $-x_{12}(n)=x_{22}(n)$.

We then hypothesized that echoes $y_{1}(n)$ and $y_{2}(n)$ can be decomposed into power series of the transmitted signals $x_{1}(n)$ and $x_{2}(n)$, respectively:

$$
\left\{\begin{array}{l}
y_{1}(n)=\sum_{i} x_{1}^{i}(n)=\left[x_{11}(n)+x_{12}(n)\right]+\left[x_{11}(n)+x_{12}(n)\right]^{2} \\
y_{2}(n)=\sum_{i} x_{2}^{i}(n)=\left[x_{21}(n)+x_{22}(n)\right]+\left[x_{21}(n)+x_{22}(n)\right]^{2} .
\end{array}\right.
$$

Note that the second order is sufficient because the transducer is not broad enough to receive the higher order.

Finally, to construct the image line $l$, the two echoes are added together:

$$
\begin{aligned}
z(n) & =y_{1}(n)+y_{2}(n) \\
& =\left[x_{11}(n)+x_{12}(n)\right]+\left[x_{11}(n)+x_{12}(n)\right]^{2}+\left[x_{21}(n)+x_{22}(n)\right]+\left[x_{21}(n)+x_{22}(n)\right]^{2} ; \\
& =\left[x_{11}(n)+x_{12}(n)\right]+\left[x_{11}(n)+x_{12}(n)\right]^{2}+\left[-x_{11}(n)-x_{12}(n)\right]+\left[-x_{11}(n)-x_{12}(n)\right]^{2} ; \\
& =2\left[x_{11}(n)+x_{12}(n)\right]^{2} \\
& =2 x_{11}^{2}(n)+4 x_{11}(n) \cdot x_{12}(n)+2 x_{12}^{2}(n),
\end{aligned}
$$

where the component $x_{11}^{2}(n)$ corresponds to the harmonic component generated at frequency $2 f_{0}$ and component $x_{12}^{2}(n)$ to the harmonic component generated at frequency $4 f_{0}$. Moreover, the intermodulation between the components $x_{11}(n) \cdot x_{12}(n)$ generates harmonic components at frequencies $2 f_{0}\left(3 f_{0}-f_{0}\right)$ and $4 f_{0}\left(3 f_{0}+f_{0}\right)$. The direct transmission of the components $x_{11}(n)$ and $x_{12}(n)$ at $f_{0}$ and $2 f_{0}$ are therefore removed from the radiofrequency line $z(n)$. Only the harmonic components from the nonlinear interaction between the excitation and the medium remain. However, since the transducer is not broad enough, the radiofrequency line $z_{k}$ is mainly composed of components $x_{11}^{2}(n)$ and $x_{11}(n) \cdot x_{12}(n)$ at $2 f_{0}$ :

$$
z(n) \approx 2 x_{11}^{2}(n)+4 x_{11}(n) \cdot x_{12}(n) .
$$




\section{Table Captions}

Table 1: Mechanical Properties of the medium explored [24]. $\mathcal{N}$ is the symbol of the Gaussian distribution. 
Table B.1:

\begin{tabular}{|c|c|c|}
\hline \multirow{3}{*}{$\begin{array}{l}\text { Tissue } 1 \\
\text { (blood) }\end{array}$} & $\rho_{1}$ & $\mathcal{N}\left(1060 \mathrm{~kg} / \mathrm{m}^{3}, 0.008 \mathrm{~kg}^{2} / \mathrm{m}^{6}\right)$ \\
\hline & $c_{1}$ & $\mathcal{N}\left(1584 \mathrm{~m} / \mathrm{s}, 0.2 \mathrm{~m}^{2} / \mathrm{s}^{2}\right)$ \\
\hline & $B / A_{1}$ & 6 \\
\hline \multirow{3}{*}{$\begin{array}{l}\text { Tissue } 2 \\
\text { (fat) }\end{array}$} & $\rho_{2}$ & $\mathcal{N}\left(928 \mathrm{~kg} / \mathrm{m}^{3}, 0.008 \mathrm{~kg}^{2} / \mathrm{m}^{6}\right)$ \\
\hline & $c_{2}$ & $\mathcal{N}\left(1430 \mathrm{~m} / \mathrm{s}, 0.2 \mathrm{~m}^{2} / \mathrm{s}^{2}\right)$ \\
\hline & $B / A_{2}$ & 10.3 \\
\hline
\end{tabular}




\section{Figure Captions}

Figure 1: Block diagram of contrast optimization in tissue pulse inversion imaging.

Figure 2: Grid of medium properties: $c_{i}$ is the wave velocity, $\rho_{i}$ the density and $B / A_{i}$ the nonlinearity parameter of the medium $i$. The ultrasound transducer was at a depth of $0 \mathrm{~mm}$, here at the top.

Figure 3: Statistics distributions of the contrast resolution optimized by a transmitted binary stochastic signal and a transmitted non-binary stochastic signal on twenty simulations. Optimizations were compared to two Gaussianmodulated sinusoidal pulses where the transmit fixed-frequency was at the optimal frequency and at two-thirds of the central frequency of the transducer.

Figure 4: (a) Distribution of the samples of the best found binary from the twenty simulations. (c) Respective transmitted waves at the transducer output (Fig. 1) when the transmitted signals were the best found binary stochastic signal $\mathbf{w}^{\star}$. (b) The respective spectra and the transducer bandwidth (black line). (e) Corresponding respective median radiofrequency line. (d) The respective spectra. As a comparison, the optimal fixed-frequency signals were depicted on red.

Figure 5: (a)Distribution of the samples of the best found non-binary stochastic signals from the twenty simulations. (c) Respective transmitted waves at the transducer output (Fig. 1) when the transmitted signals were the best found non-binary stochastic signal $\mathbf{w}^{\star}$. (b) The respective spectra and the transducer bandwidth (black line). (e) Corresponding respective median radiofrequency line. (d) The respective spectra. As a comparison, the optimal fixedfrequency signals were depicted on red.

Figure 6: Simulation of short-term harmonic powers computed on the radiofrequency line when the transmitted signal was the best found non-binary stochastic command, the best found binary stochastic command, a Gaussian-modulated sinusoidal pulse at the optimal transmit fixed-frequency and a Gaussianmodulated sinusoidal pulse at the usual transmit fixed-frequency $\left(2 / 3 f_{c}\right)$. The statistical distribution of the harmonic short-term mean powers for the twenty simulations are depicted by areas (first quartile, second quartile, median, third quartile and fourth quartile).

Figure 7: Synthetic images using (a) the usual transmitted signal at two-thirds of the central fixed-frequency of the transducer $2 / 3 f_{c}$, (b) the usual transmitted signal at the optimal transmit fixed-frequency, (c) the best found binary stochastic signal and (d) the best found non-binary stochastic signal. The images showed the medium depicted in Fig. 2.

Figure 8: Block diagram of the experimental setup.

Figure 9: (a) Experiment of the contrast resolution optimization by a transmitted binary stochastic signal and a transmitted non-binary stochastic signal. Optimizations were compared to two Gaussian-modulated sinusoidal pulses where the transmit fixed-frequency was at the optimal frequency and at two-thirds 
of the central frequency of the transducer. (b) and (c) best found binary and non-binary stochastic signal $\mathbf{w}^{\star}$ respectively, the signal in opposite and the difference. (d) and (g) Respective transmitted waves at the transducer output. (e) and (h) Corresponding radiofrequency lines. (e) Their respective short-term harmonic powers. (f) and (i) The spectra of the transmitted and backscattered waves transmitted by using best found binary and non-binary stochastic signal respectively and the transducer bandwidth (black line).

Figure 10: Experimental images using (a) the usual transmitted signal at two-thirds of the central fixed-frequency of the transducer $2 / 3 f_{c}$, (b) the usual transmitted signal at the optimal transmit fixed-frequency, (c) the best found binary stochastic signal and (d) the best found non-binary stochastic signal. 


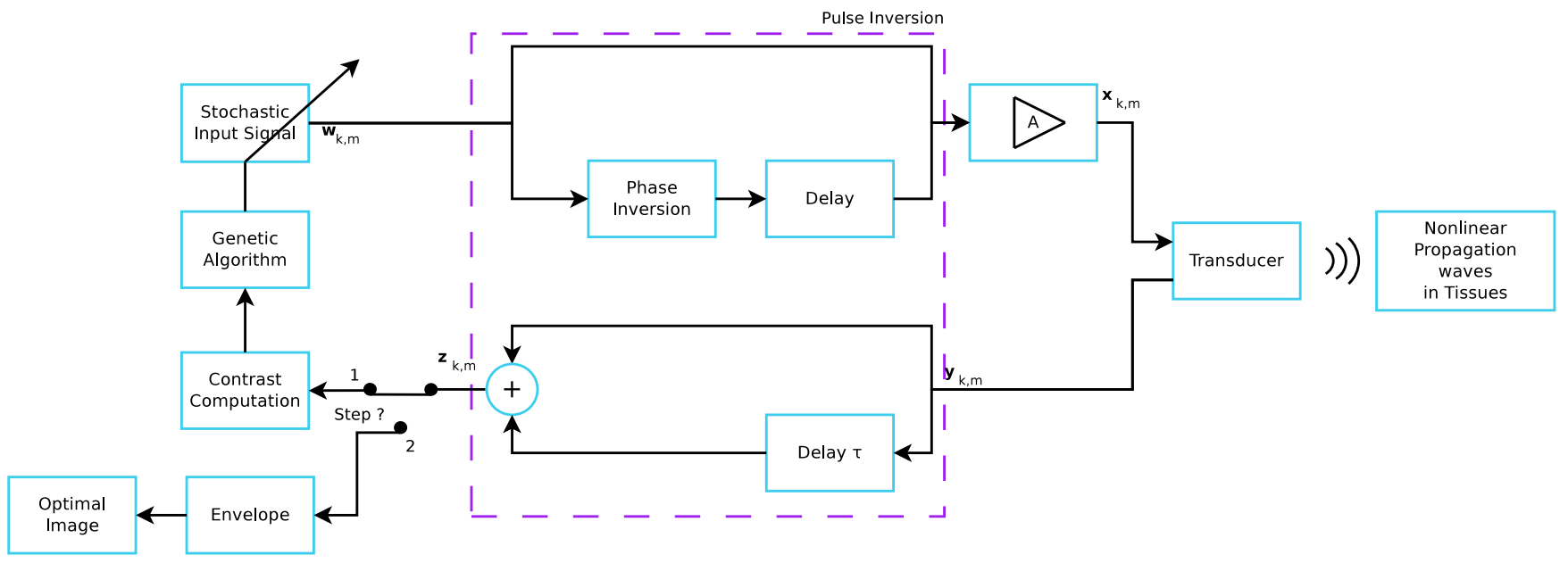

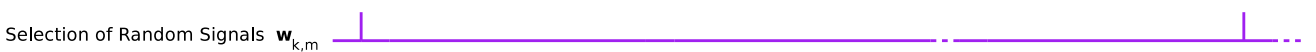

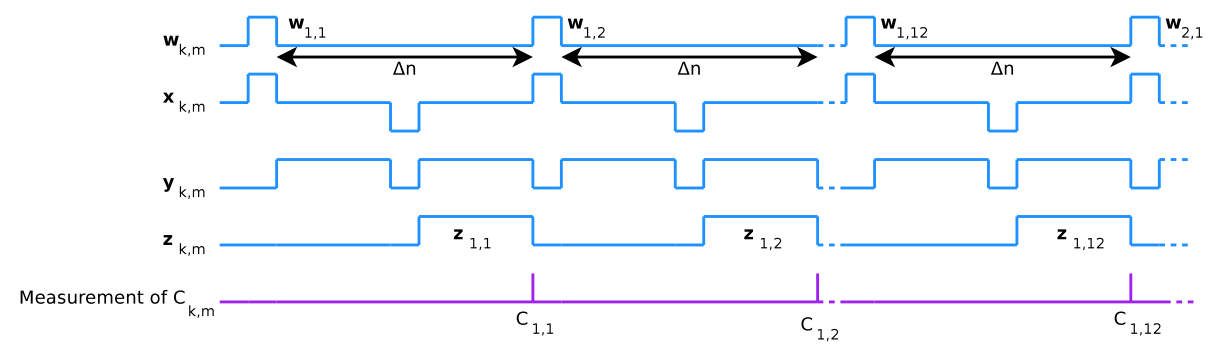

Figure 1 


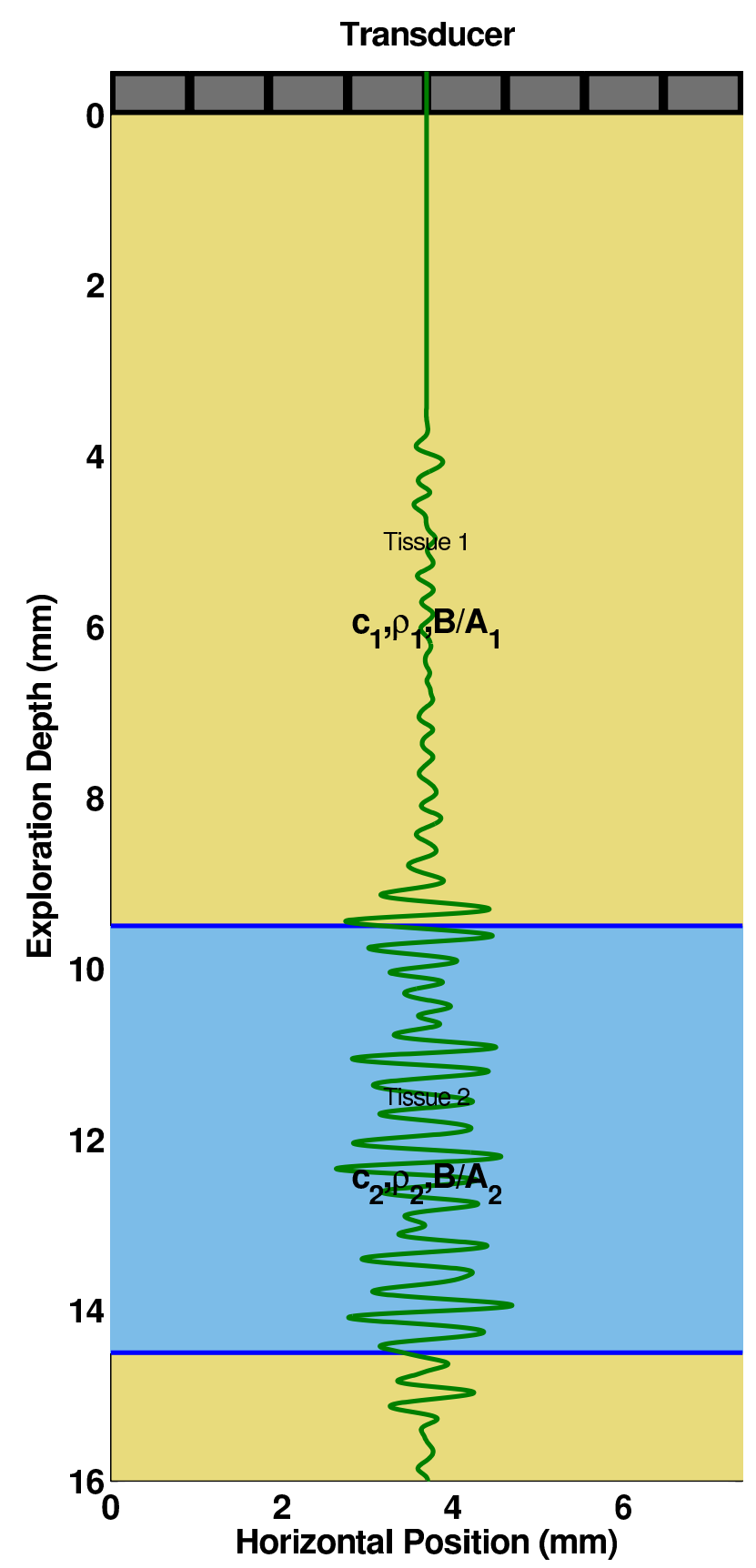

Figure 2 


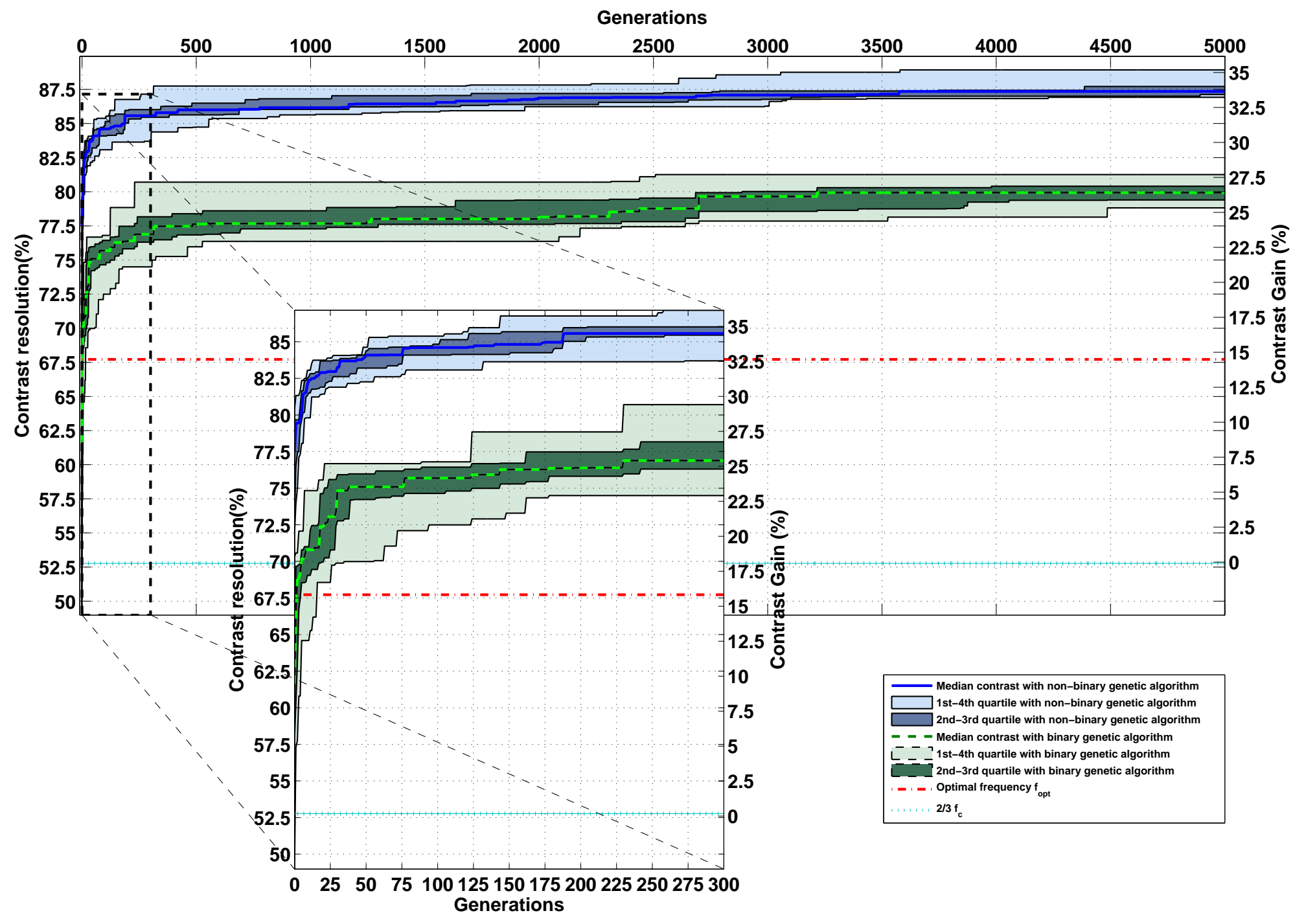

Figure 3 


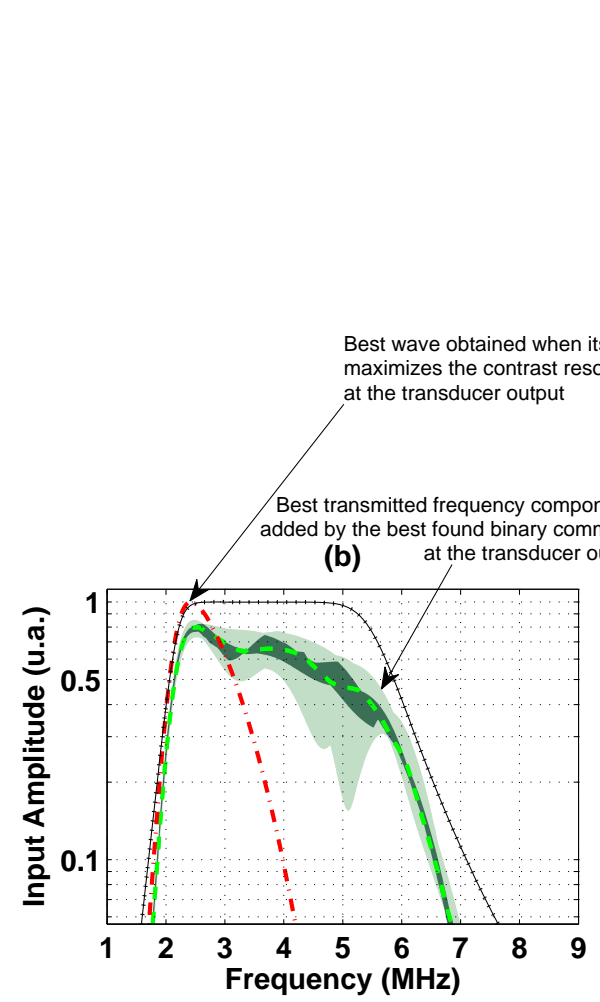

(d)

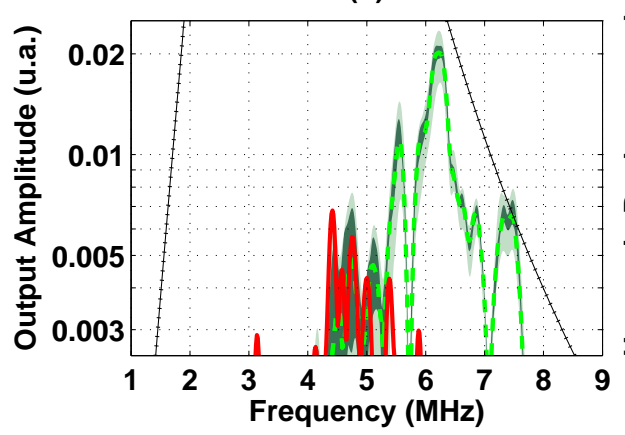

(a) Binary Stochastic Commands
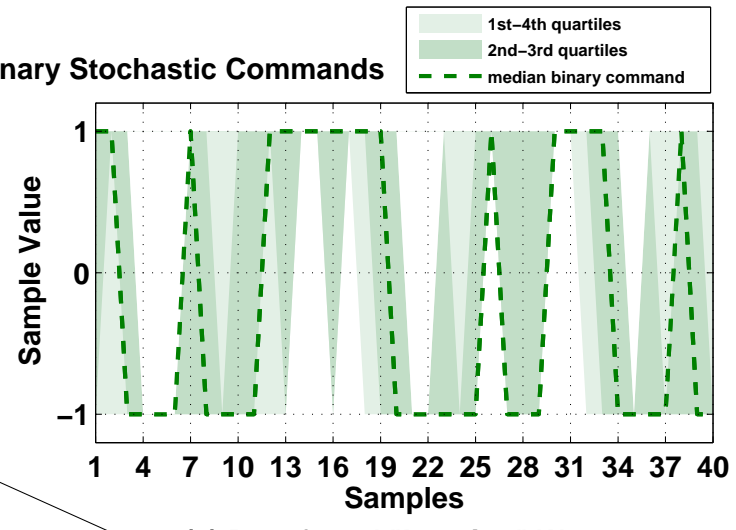

(c) Best found "Imaging" Waves

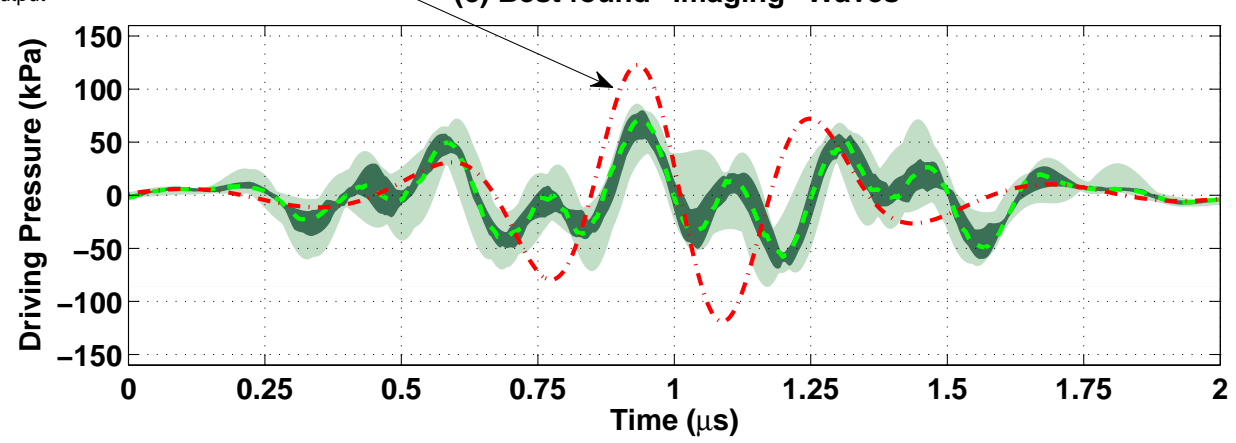

(e) Radiofrequency Lines

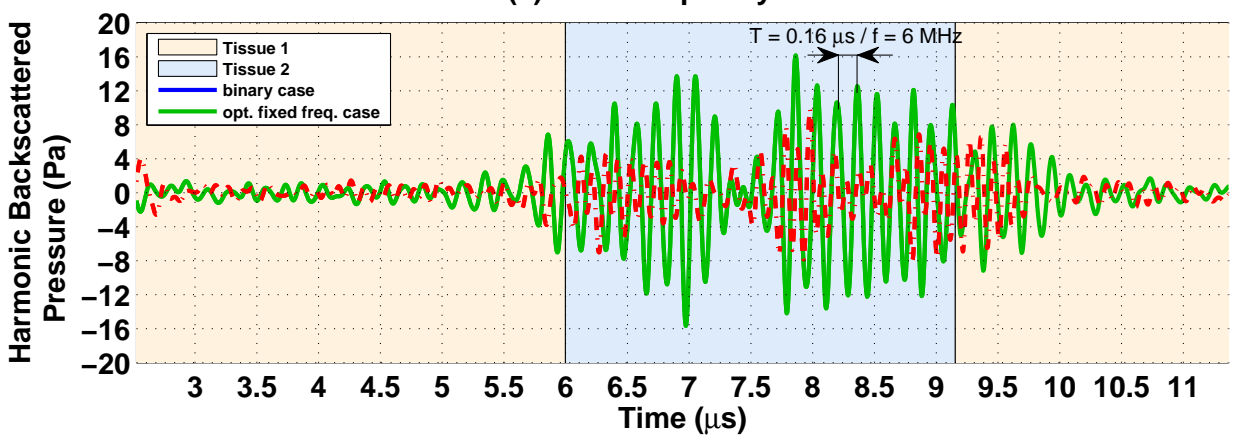

Figure 4 


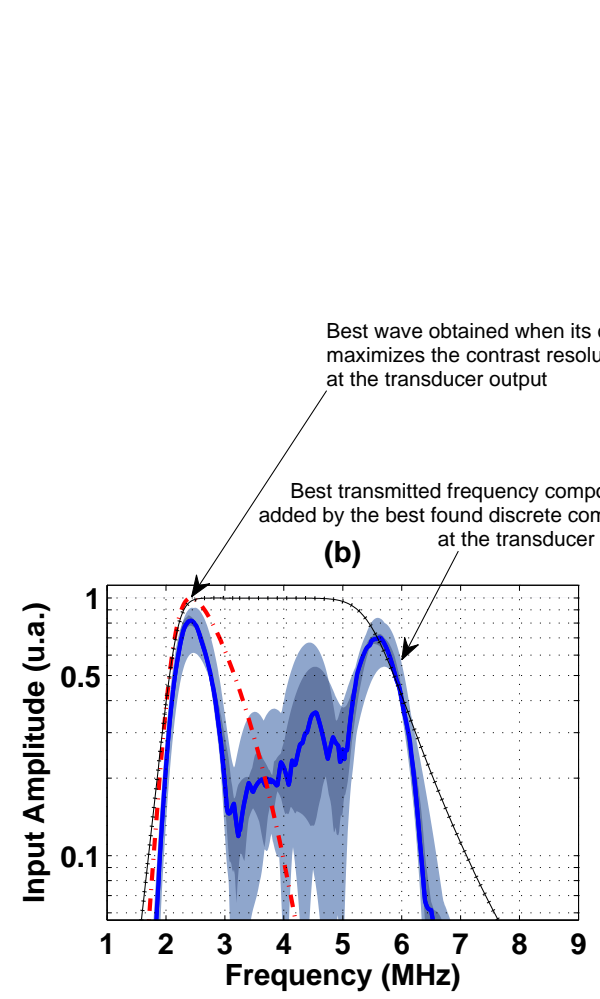

(d)

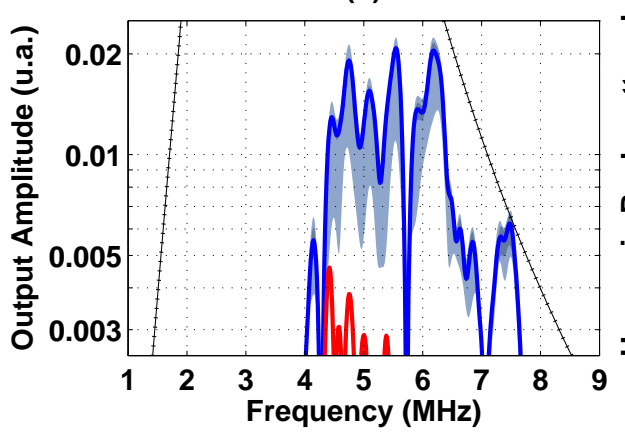

(a) Non-binary Stochatic Commands \begin{tabular}{l}
$\begin{array}{l}\text { 1st-4th quartiles } \\
\text { 2nd-3rd quartiles } \\
\\
\end{array}$ median discrete command \\
\hline
\end{tabular}

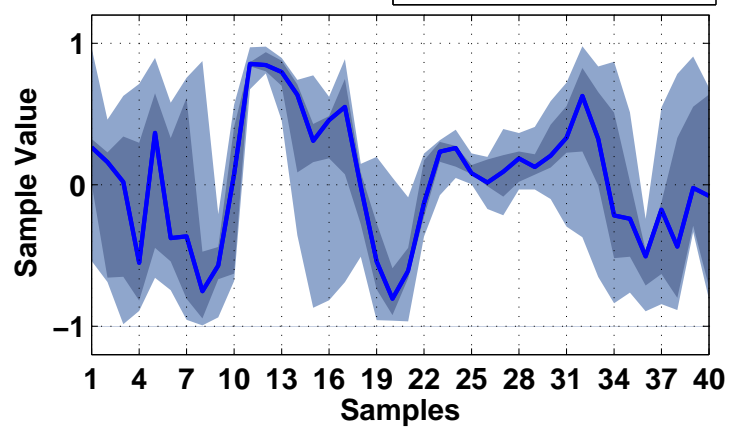

(c) Best found "Imaging" Waves

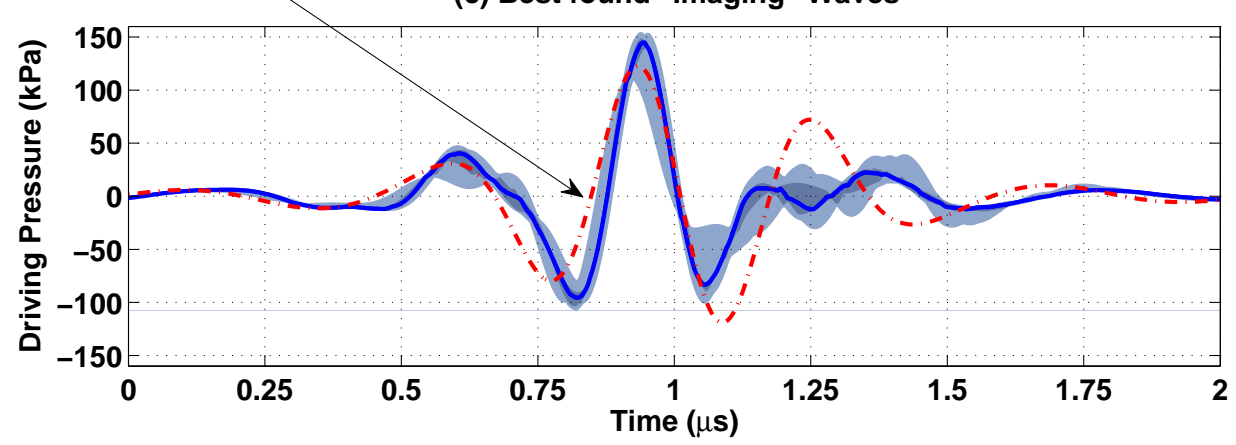

(e) Radiofrequency Lines

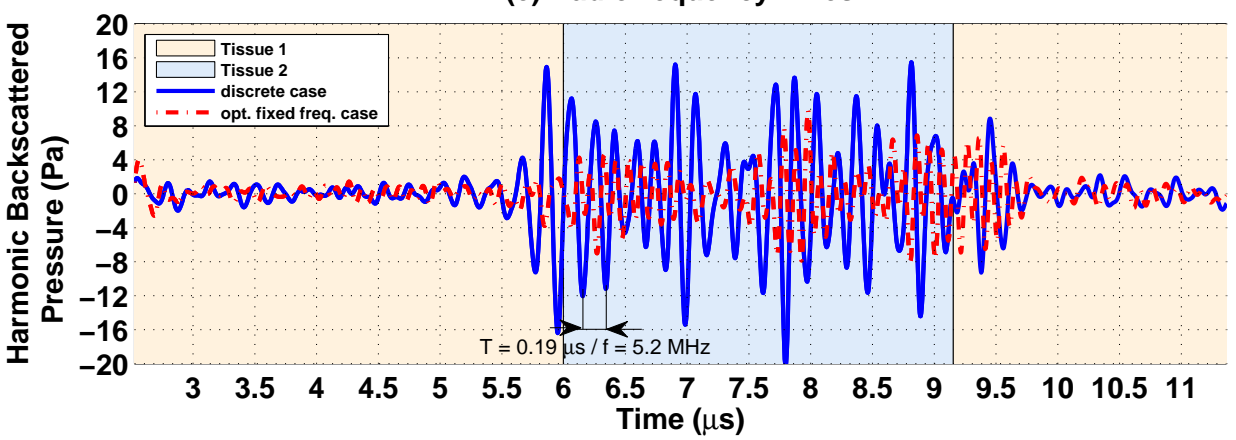

Figure 5 


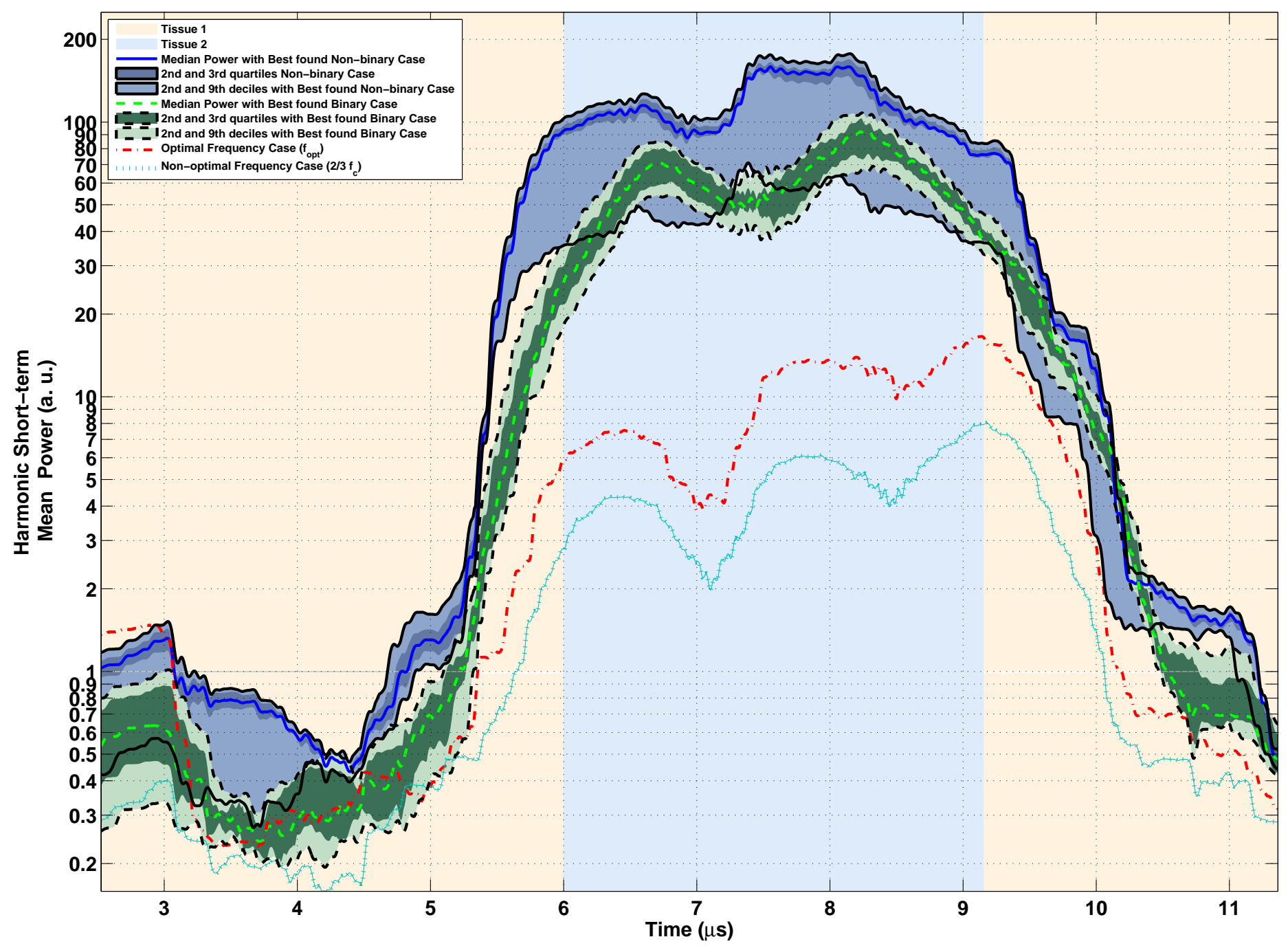

Figure 6 
(a) $C=53 \%$

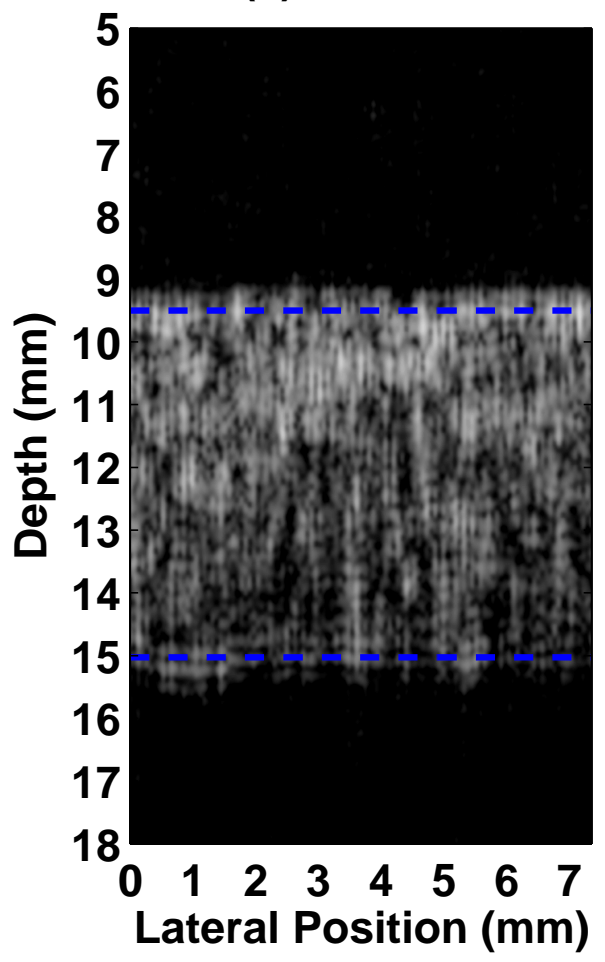

(c) $79 \%$

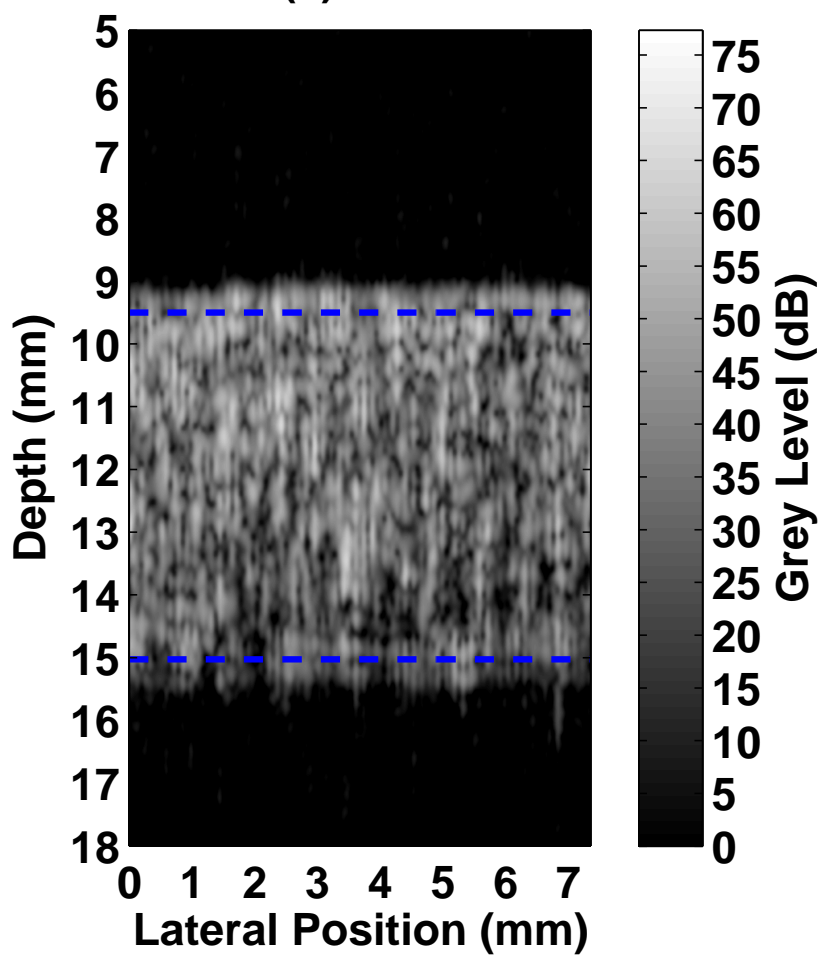

(b) $C=72 \%$

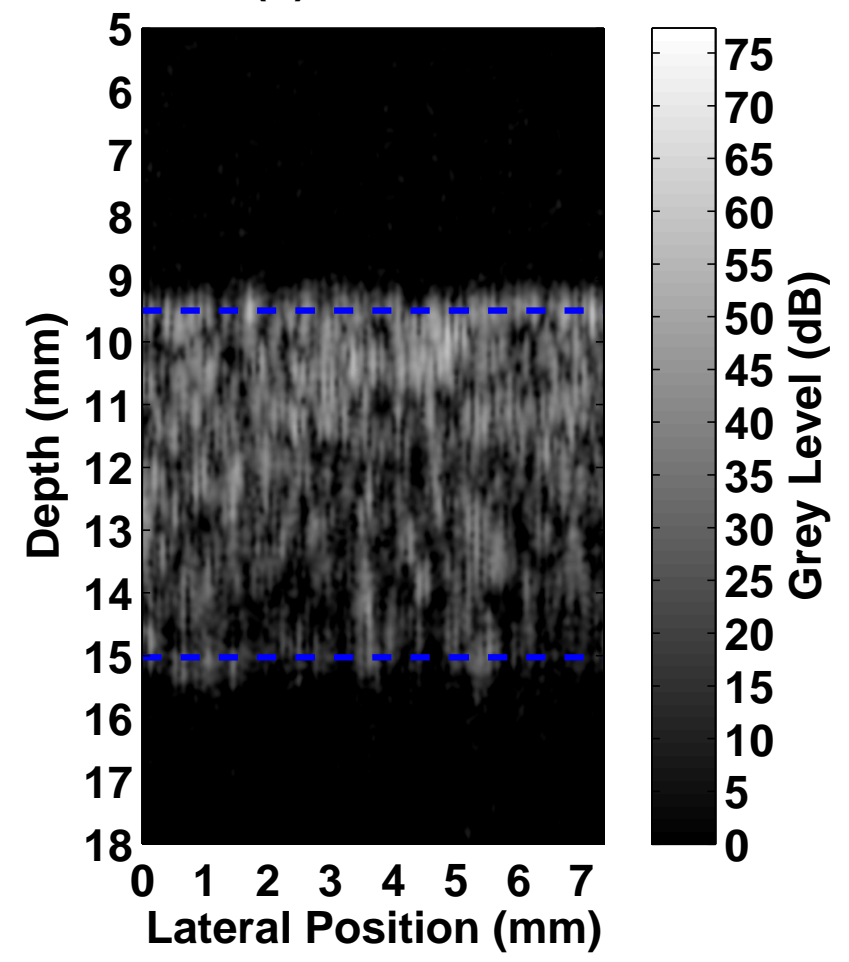

(d) $85 \%$

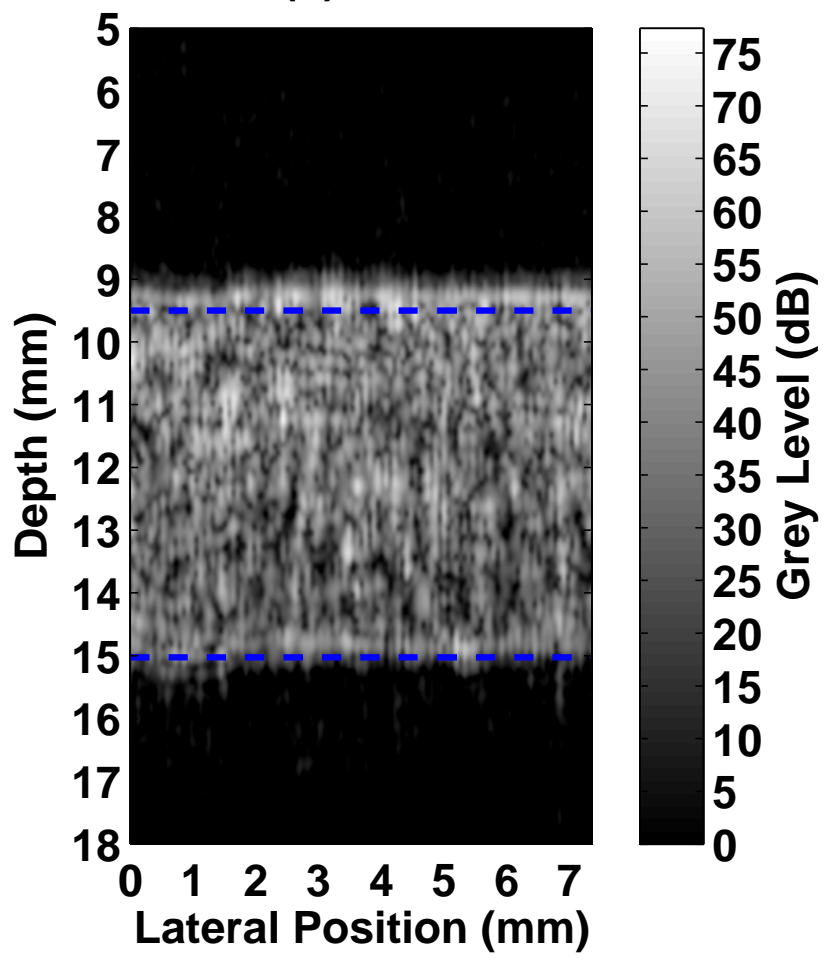




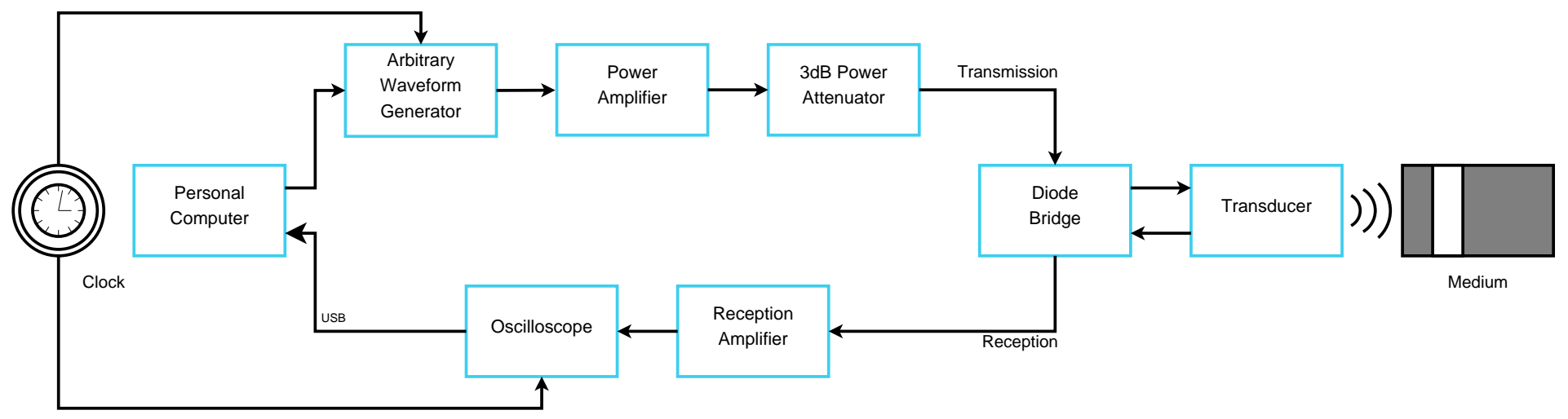

Figure 8 

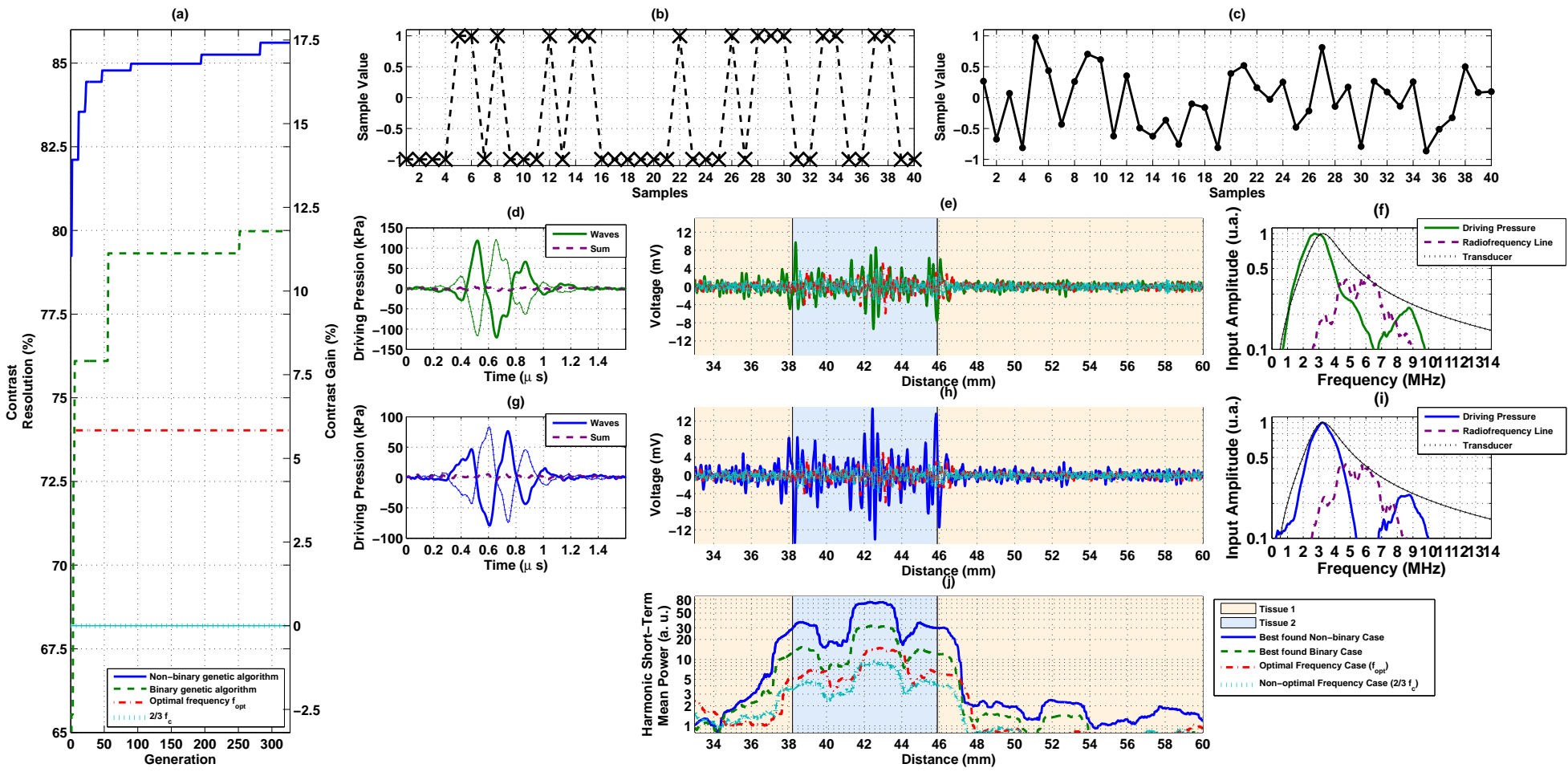

Figure 9 
(a) $\mathrm{C}=65 \%$

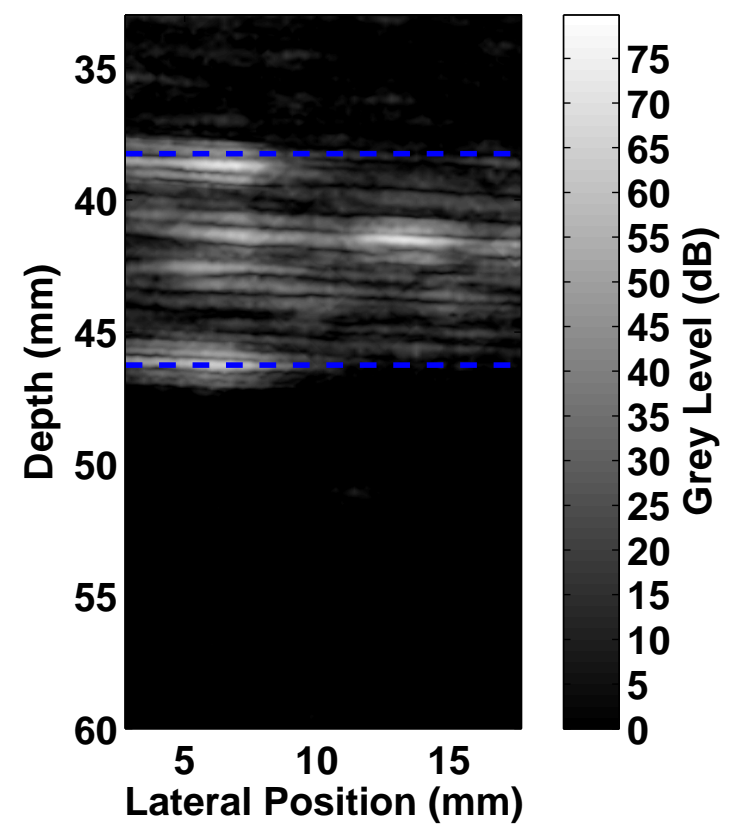

(c) $\mathrm{C}=79 \%$

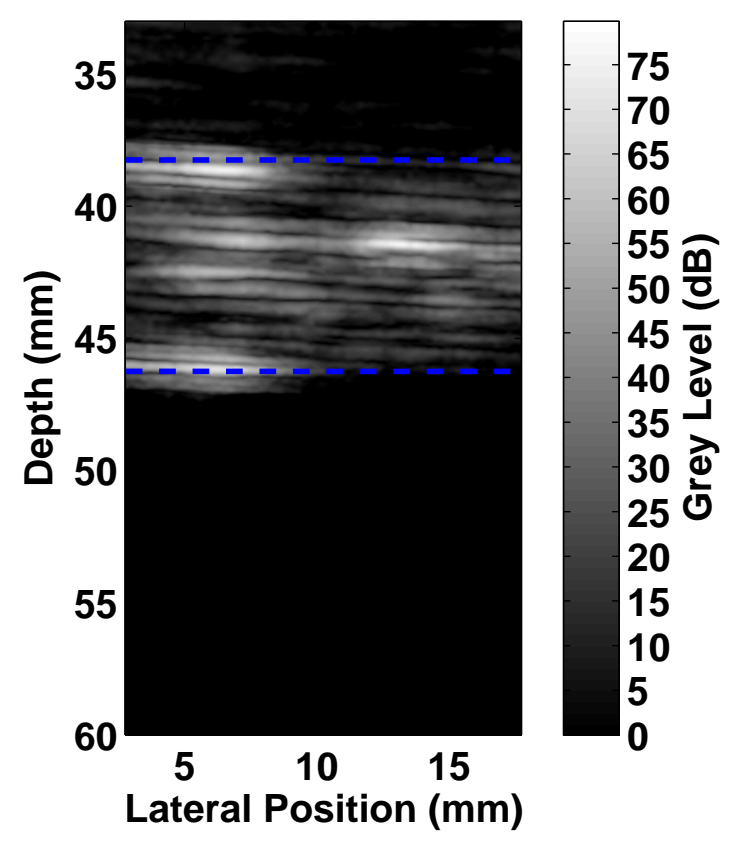

(b) $\mathrm{C}=70 \%$

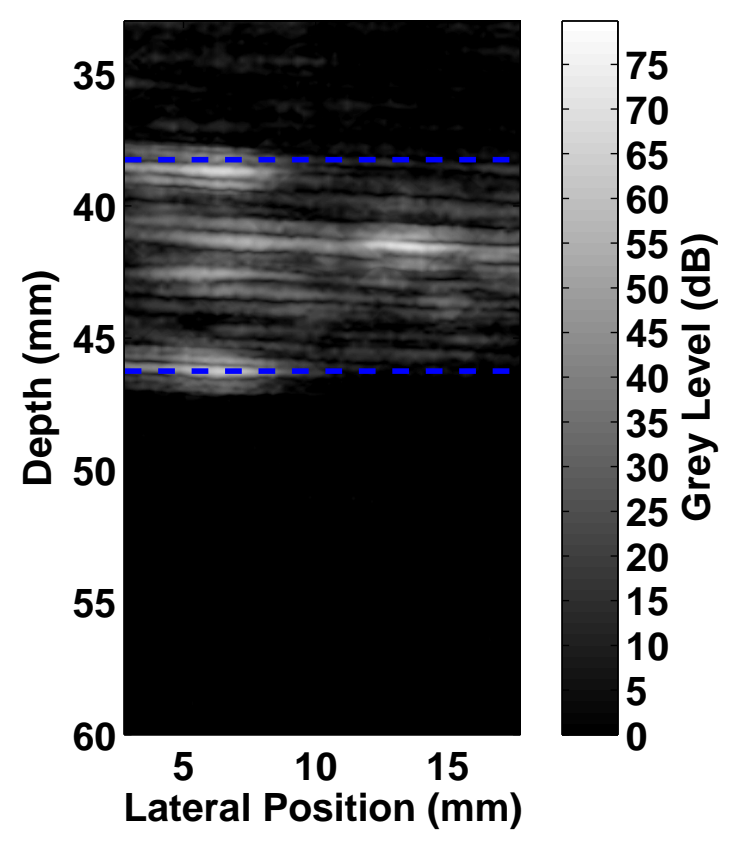

(d) $\mathrm{C}=83 \%$

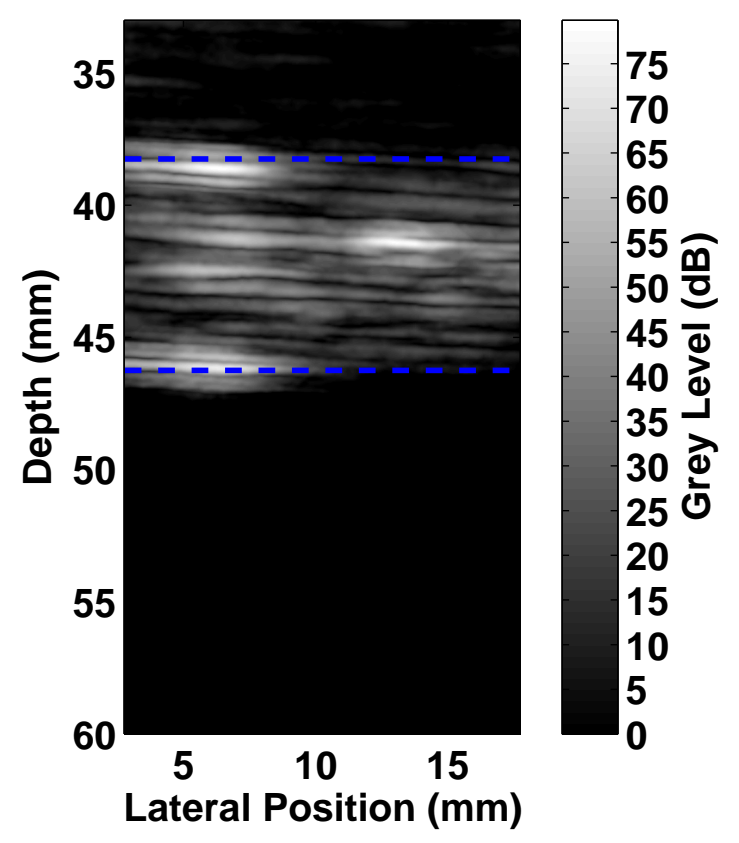

